\title{
Information-Based Manufacturing Program Summary and Accomplishments
}

A NIST program promoting collaborative research and developing competence in the use of advanced information technology for research, standards and measurement services.

\section{David C. Stieren Mark E. Luce}

U.S. DEPARTMENT OF COMMERCE Technology Administration Manufacturing Engineering Laboratory Office of Mariufacturing Programs National Institute of Stanclards and Technology

Gaithersiourg, MD 20899

ac

100

.456

10.6490

2000

C. 2

\section{NGT}

National Instifute of Standards and Teshnology

Technology Administration

U.S. Department of Commerce 



\section{Information-Based Manufacturing Program Summary and Accomplishments}

A NIST progran pronoting collaborative research and developing competence in the use of advanced information technology for research, standards and measurenent services.

David C. Stieren

Mark E. Luce

U.S. DEPARTMENT OF COMMERCE

Technology Administration

National Institute of Standards and Technology

Manufacturing Engineering Laboratory

Office of Manufacturing Programs

Gaithersburg, MD 20899

March 2000

U.S. DEPARTMENT OF COMMERCE

William M. Daley, Secretary

TECHNOLOGY ADMINISTRATION

Cheryl L. Shavers, Under Secretary for Technology

NATIONAL INSTITUTE OF STANDARDS AND TECHNOLOGY

Raymond G. Kammer, Director 


\title{
Office of Manufacturing Programs
}

The Office of Manufacturing Programs (OMP) manages cross-division and crosslaboratory, collaborative research and development (R\&D) programs within the Manufacturing Engineering Laboratory of the National Institute of Standards and Technology (NIST), including the Information-Based Manufacturing Program. The Information-Based Manufacturing Program consists of sixteen collaborative research projects distributed among the seven NIST measurement and standards laboratories, as well as the Manufacturing Extension Partnership. The OMP manages these projects by tracking deliverables, publishing program progress reports, conducting management reviews of technical activities and funding obligations, planning and conducting technology demonstrations, and preparing program descriptive materials for public dissemination.

The points of contact within OMP for the Information-Based Manufacturing Program are:

\author{
Mark Luce, Chief, OMP \\ mark.luce@nist.gov \\ David Stieren, Program Manager \\ david.stieren@.nist.gov
}

\section{Disclaimer}

No approval or endorsement of any commercial product by the National Institute of Standards and Technology is intended or implied. Certain commercial equipment, instruments, or materials are identified in this report in order to facilitate understanding. Such identification does not imply recommendation or endorsement by the National Institute of Standards and Technology, nor does it imply that the materials or equipment identified are necessarily the best available for the purpose.

This publication was prepared by United States Government employees as part of their official duties and is, therefore, a work of the U.S. Government and not subject to copyright. 


\section{Acknowledgements}

The following NIST staff members have contributed to the project summary information contained in this document's Appendix:

Dr. E. Clayton Teague

Dr. Theodore Vorburger

Dr. Alkan Donmez

Dr. Johannes Soons

Albert Wavering

Neil Christopher

Dr. William Stone

Eric Steel

Dr. Dale Newbury

Dr. Michael Postek

Dr. Timothy Foecke
Dr. Christopher Soares

Stephen Seltzer

Barbara Goldstein

Dr. George Kelly

Dr. Pedro Espina

Nile Oldham

Douglas Montgomery

Stephen Thompson

Timothy Quinn

Dr. Marc Desrosiers

Additional information about NIST, including the NIST Manufacturing Engineering Laboratory and the program information described in this report, is available on the World Wide Web at the following addresses:

http://wnw.nist.gov

http://www.nist.gov/mel 


\section{Table of Contents}

Introduction .................................................... 1

Program Overview................................................ 1

National Advanced Manufacturing Testbed ................. 3

Program Accomplishments ...................................... 5

Measures of Program Success............................... 13

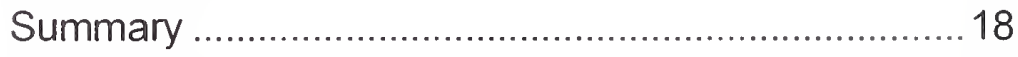

Appendix: Project Summaries ...............................20

References ..................................................... 51 


\section{INTRODUCTION}

The Information-Based Manufacturing Program of the National Institute of Standards and Technology (NIST) is developing competence across NIST in the use of information technology (IT) to aid in the conduct of research, accelerate the development of standards, and improve the delivery of NIST measurement and testing services, especially with respect to manufacturing.

The purpose of this report is to summarize the Information-Based Manufacturing Program by providing an overview of the program and highlighting key accomplishments that have been achieved. The appendices provide additional information that describes the technical projects that constitute the program's research portfolio.

The intended audience for this document includes NIST staff working on the sixteen projects of the Information-Based Manufacturing Program, private sector organizations collaborating with NIST, and the industrial public in general. This report is available on the World Wide Web via the home page of the NIST Manufacturing Engineering Laboratory (MEL), which is located at http://unww.nist.gov/mel.

Copies of this report can also be obtained via electronic mail request to the points of contact from the MEL Office of Manufacturing Programs identified on page (ii) of the report.

\section{PROGRAM OVERVIEW}

The NIST Manufacturing Engineering Laboratory (MEL) initiated the InformationBased Manufacturing Program in 1996 to enhance the development of informationbased technologies and standards for U.S. manufacturing industries, specifically those working in the discrete-part, mechanical manufacturing sectors. The program was also initiated to improve MEL's ability to deliver measurement and testing services for industry.

Initially, MEL designed the program's strategy to promote collaborative research across the various MEL Divisions and to develop competence in the use and exploitation of IT for research and measurements and standards activities throughout MEL. As the program evolved, the strategy changed to encompass collaborations with the other NIST Measurement and Standards laboratories. This evolution expanded the technical focus to include technologies, measurements, and standards aimed at industrial sectors being served by the other NIST laboratories, and not just MEL. 
During the program, competence has been developed across all seven NIST Laboratories and the Manufacturing Extension Partnership (MEP) Program to exploit IT in the improvement of

1. the conduct of manufacturing research performed by NIST for and with American industry

2. the delivery of NIST measurement services

3. the means by which NIST scientists and engineers access the tools and information they need to do their jobs, including equipment, software, other NIST scientists, and non-NIST collaborators

This program began with a set of four inter-division MEL projects. Fueled with additional financial support from the NIST Director, the program matured into a collection of sixteen inter-laboratory projects that exploit the capabilities of highperformance networking and advanced computing technologies. Each of the sixteen projects derives its technical and standards basis from specific core programs and initiatives contained within distinct NIST laboratories. Additional information about the activities of these projects can be found in the Appendix.

The Information-Based Manufacturing program has provided these projects with an advanced information infrastructure enabling access to a collaborative, testbed environment. In this environment, multidisciplinary expertise is leveraged through cross-lab participation. These elements have combined to put NIST resources online across the agency, allowing significant amounts of hardware, software, and staff expertise to be accessible by both NIST customers and NIST scientists. This has successfully promoted the effective and efficient application of these resources to the collaborative conduct of research and the improved delivery of measurement and testing services.

With MEL providing technical and organizational support to all of the projects, the program has demonstrated successfully the beginning of a new era of crosslaboratory cooperation at NIST. The focus on incorporating IT into projects to leverage resources and foster inter-disciplinary collaboration has significantly improved the way NIST scientists and engineers serve American industry. Furthermore, the program has clearly and successfully demonstrated that NIST is an agency that is fully embracing leading-edge IT to execute its congressionallymandated mission. The NIST mission is to foster sustained U.S. economic growth by working with industry to develop and apply technology, measurements, and standards. 


\section{NATIONAL ADVANCED MANUFACTURING TESTBED}

The primary supporting resource of the Information-Based Manufacturing Program has been the National Advanced Manufacturing Testbed (NAMT)[1]. The NAMT is a physical testbed built on a state-of-the-art, high-speed computing and communications infrastructure. The NAMT enables scientists and engineers from industry, NIST, academia, and government agencies to work collaboratively to solve measurement and standards issues that impede companies and industries from making the most of their IT.

The NAMT provides network connectivity and computing support to the projects of the Information-Based Manufacturing Program. Projects use this support to demonstrate how leading-edge IT is being exploited in the performance of advanced research, the development of standards, and the development and improved delivery of NIST measurement services. The NAMT provides project participants the ability to connect their research laboratories, equipment, and software into a high-performance network backbone. This allows the simultaneous communication of voice, video, and data information over a single physical medium to occur at high speeds and at guaranteed qualities of service.

The network connectivity requirements of the Information-Based Manufacturing Program frequently exceed what is available using the existing Internet. The NAMT network backbone allows, for example, live video signals to be transmitted in fullmotion, 30 frames-per-second capacity between connected laboratory sites. Video, audio, and data can be communicated with negligible latency and with guaranteed quality of service. This basically means that if a communication session contains the transfer of information requiring some minimum amount of bandwidth, the NAMT network backbone technology is capable of providing that bandwidth without regard to any contention issues. Contention issues are typical of multiple-user, broadcast environments with low bandwidth transmission limitations.

The NAMT network backbone is also flexible enough to accommodate electronic collaboration that is based upon simple Internet connections. This is important because high-performance networking represents the next generation of capabilities for today's Internet. Not all partners in the Information-Based Manufacturing Program utilize sophisticated, high-performance networking, and connections with these partners must occur with today's commodity Internet.

Each of the sixteen research projects in the program utilizes the NAMT as an "Information Age workbench." The projects test new technologies and showcase how machines, software, and people can be networked together, efficiently and effectively, to improve productivity and foster innovation.

The NAMT supports geographically distributed partners and it provides the means for projects to utilize virtual technology (i.e., modeling and simulation) in support of 
advanced research. Equipment, expertise, and other capabilities of program partners are connected electronically through the NAMT computing and communications infrastructure. Moreover, collections of resources and personnel can be easily configured - and reconfigured - in response to changing research needs and objectives.

The collaborative R\&D environment supported by the NAMT is similar in many ways to the concepts of a collaboratory, a term derived from a 1993 National Research Council (NRC) Report [2]. A collaboratory can be considered as a "research center without walls." Within the NAMT collaboratory, various tools and systems that use computing and networking technology to aid manufacturing research are integrated together. This provides an environment that allows scientists and engineers to make more efficient use of manufacturing resources, regardless of where they are located. The NAMT collaboratory structure is shown schematically in Figure 1.

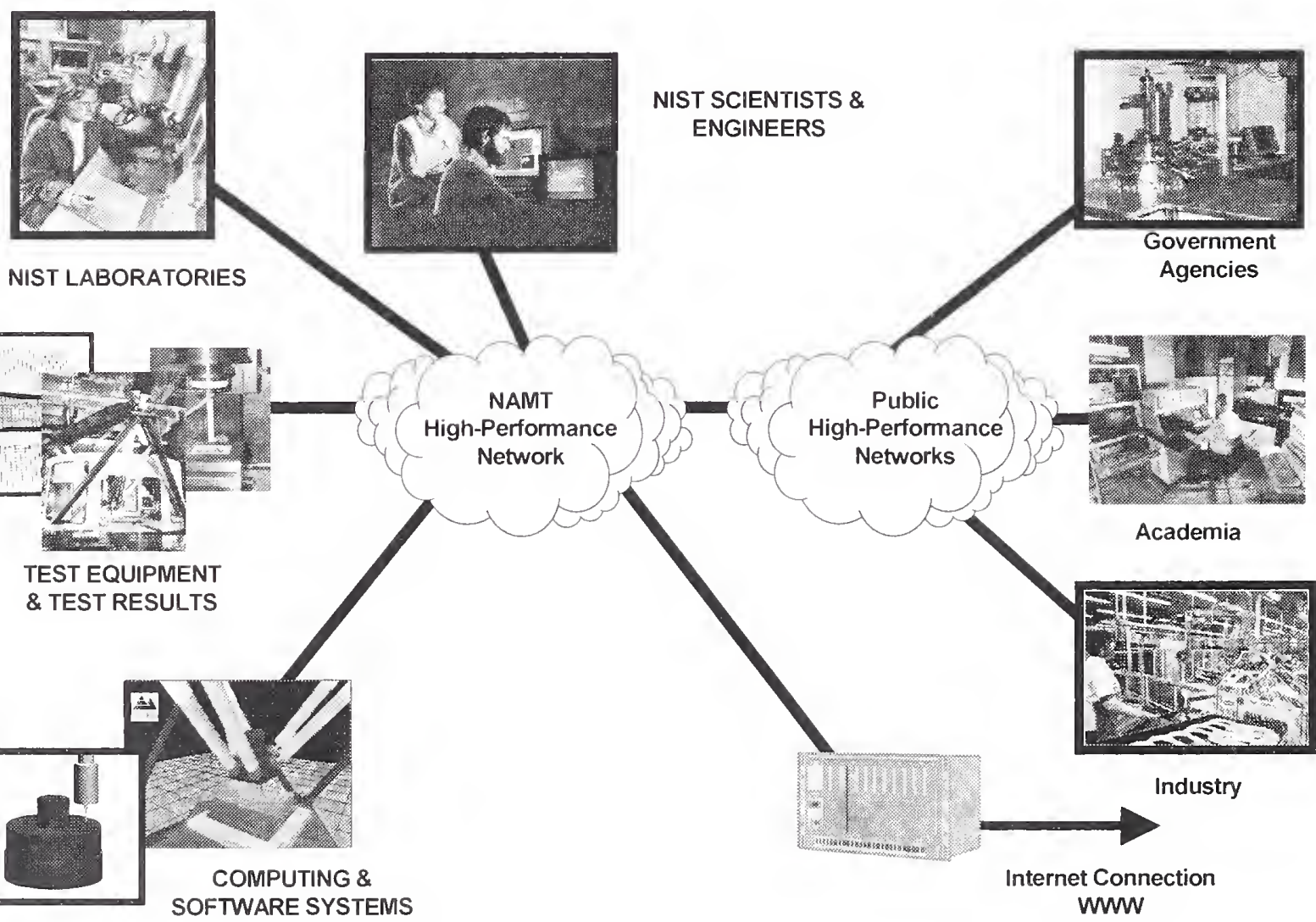

Figure 1. The NAMT Collaboratory 
The NAMT supports research on applications of simulation and modeling methods that are essential to rapid and predictable production, among a host of other key capabilities. Information-Based Manufacturing Program research supported by the NAMT develops and validates models and simulations of individual processes, as well as entire manufacturing systems.

The NAMT has created a significant opportunity to pool resources and capabilities, to share risks, and, most important, to efficiently build the technical underpinnings of an advanced information infrastructure required by American manufacturing industries. Such a foundation and infusion of state-of-the-art computing and communications technologies is enabling MEL and other NIST laboratories to address issues that affect multiple manufacturing sectors. The NAMT is being used by the InformationBased Manufacturing Program as a platform for experimenting with technology and standards solutions that American manufacturing industries will encounter as they compete in the emerging digital economy of the $21^{\text {st }}$ century.

\section{PROGRAM ACCOMPLISHMENTS}

Since 1996, the Information-Based Manufacturing program has been operating a successful strategy to promote collaborative research across NIST and develop competence in the use and exploitation of advanced information technologies. New levels of technical competence have been achieved in the aforementioned areas, and these competencies are the primary results that validate the success of that strategy.

The following summarizes the capabilities and competencies that have been developed as a result of the program. Specific instances of how these have been created by the program are highlighted.

\section{The ability to deliver NIST measurement and testing services on-line}

This has been demonstrated by incorporating telepresence capabilities and network laboratory linkages for:

- tele-calibration of gas flow meters and standards

- remote, collaborative measurement of electrical standards, specifically digital multi-meters

- traceable, collaborative, physical and chemical analysis of standard reference artifacts performed using scanning tunneling, scanning electron, tunneling electron, and analytical electron microscopes

- dosimetry calibrations and standards for radiation sources used in medical procedures, including certain treatments of cancer, and for radiation processing industries 
One example of a new measurement and testing service made possible by this program was the successful deployment of a pilot network to enable collaboration among measurement laboratories in eleven nations. This pilot is called SIMnet, an internet-based network intended to speed development of the emerging Interamerican Metrology System, or SIM [3].

Through SIMnet, NIST and 10 other National Measurement Institutes (NMIs) are now able to participate remotely in "round robin" measurement comparisons. These provide the technical basis for ensuring confidence in the measurement capabilities of trading partners. Typically, multinational comparisons take several years and involve personnel from the lead laboratory traveling from country to country. These personnel provide expertise and training to assist in setting up the carefully controlled conditions necessary for valid comparisons of measurements. Initiated as a pilot in 1999, SIMnet is demonstrating significant reductions in time and cost involved in achieving measurement comparability and traceability throughout the Americas.

By means of the Internet, personal computers, and inexpensive videoconferencing software and hardware, NMI staff in all participating countries will be able to observe personnel in another nation as they perform a measurement comparison. They also will be able to review the data as it is collected, ask questions, and serve as consultants to the NMI performing the measurements.

The Organization for American States inaugurated this NIST-led effort to help to ensure that measurements made in one American nation can be compared directly to those made in another. Measurement comparability is an essential infrastructural component of the planned Free Trade Area of the Americas, envisioned to extend from Alaska to Tierra del Fuego by the year 2005.

Another example of Information-Based Manufacturing program success comes from a project that was begun in 1998. The Tele-Calibration of Gas Flow Meters project, led by the NIST Chemical Science and Technology Laboratory (CSTL), is focused on the improvement of a specific set of NIST measurement services. The project seeks to change the paradigm used to deliver flow-rate calibration services throughout the Nation by using tele-presence to create a Calibration-Web capable of

- leveraging the expanded flow-rate capabilities of US secondary flow laboratories in support of NIST's standards business

- providing remote in-situ calibrations of flow meters to US industry

- enabling the remote monitoring of laboratories accredited by the NIST National Voluntary Laboratory Accreditation Program

- allowing tele-comparisons of national standards among National Metrology Institutes worldwide

This project has enabled NIST to be in a position to serve an expanded range of flow calibration requirements of U.S. industry. By using telepresence and forging critical industrial partnerships, technology is enabling NIST scientists to expand the range of 
NIST gas flow calibrations by making the facilities at an industrial calibration laboratory in Nunn, CO, available for use in NIST calibration operations over a widearea high-performance computing and communications network. By installing NIST instrumentation at the industrial facility, this instrumentation can be accessed using Web-based technology by NIST personnel to provide NIST-certified calibrations outside NIST's Gaithersburg campus.

This project has, therefore, allowed NIST to improve the means by which it delivers gas flow calibrations by expanding the range of flow calibration capabilities that NIST can provide. Exploitation of IT, coupled with industrial partnerships, has allowed this to happen without NIST incurring the several million-dollar costs associated with upgrading its own facilities in Gaithersburg.

The provision of remote access to NIST equipment, instrumentation and systems used in advanced manufacturing and measurement research

This has been demonstrated by providing network linkages to NIST research labs for the communication of voice, video, and data information to and from those labs as a means of improving organizational ability to leverage resources. This has included the ability in many instances to tele-operate the research platforms in these labs. This basically means that NIST research labs, and the equipment, instrumentation, systems and scientists in them, are now accessible in real time from remote locations. This facilitates the performance of research between geographically distributed partners as effectively and efficiently as if the partners were physically colocated with one another.

The NIST labs and facilities possessing and using these capabilities are listed below. The NIST Organizational Unit $(\mathrm{OU})$ that operates each lab and facility is also included.

- the Octahedral Hexapod machine and strut test lab (MEL)

- the Monarch Metalist turning machine lab (MEL)

- the Automated Welding Manufacturing System in Gaithersburg (MEL), and the weld analysis lab in Boulder (Materials Science and Engineering Laboratory (MSEL))

- the tensile testing and analysis lab (MSEL)

- advanced analytical microscope systems (MEL and Computer Science and Technology Laboratory (CSTL))

- gas flow measurement systems and facilities (CSTL)

- electrical standards calibration instrumentation (Electronics and Electrical Engineering Laboratory (EEEL))

- the Next Generation Inspection System lab (MEL )

- the TETRA and RoboCrane robotic systems (MEL ) 
- the Computer Integrated Construction Environment lab and National Construction Automation Testbed (Building and Fire Research Laboratory (BFRL))

- dosimetry measurement systems (Physics Laboratory (PL))

- network protocol and collaborative software tool development and testing labs (Information Technology Laboratory (ITL))

For example, one of the program's original projects is exploiting IT to develop and disseminate an understanding of the characteristics and capabilities of a revolutionary new class of machine tools. This Characterization, Remote Access, and Simulation of Hexapod Machines project is examining a promising new technology that marks the first major departure in machine tool design in nearly a century. By providing remote access to its experimental Octahedral Hexapod Machine, NIST is helping a national group of machine tool builders, prospective users, and researchers build experience with and evaluate the capabilities of this type of machine (see Figure 2 below). For hexapods to achieve widespread use, a number of important questions and issues must be resolved. Through this project, NIST is helping remotely located industry and university researchers investigate the performance characteristics and capabilities of the experimental technology. The project is also developing and using new simulation and modeling tools, as well as examining critical control and integration issues.

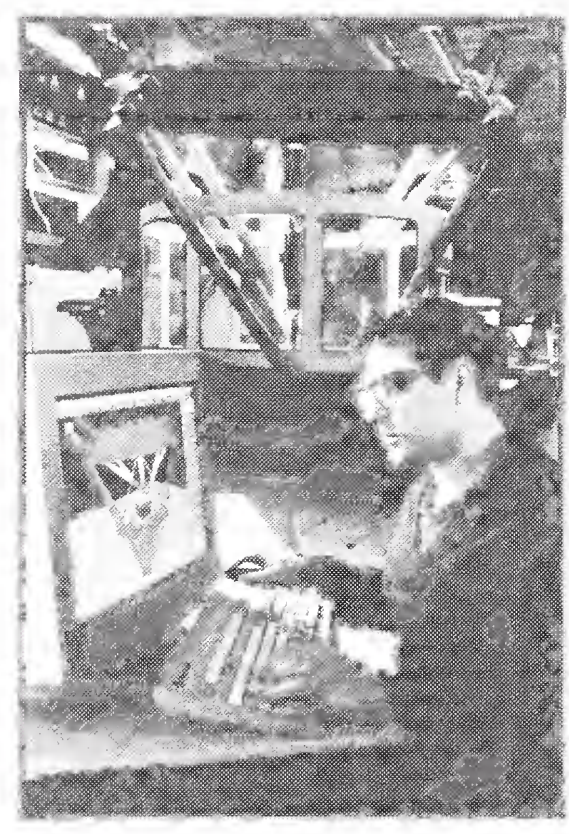

Figure 2. NIST Octahedral Hexapod Machine

Currently, real-time hexapod collaborations primarily involve "over the shoulder" participation, enabled by cameras manipulated by off-site observers. More extensive capabilities for remote collaboration, including the sharing of hexapod sensor and 
control information, are also being investigated. They are likely to become integral to research as widespread advances in communication, networking, and security technologies occur. Characterizing and enhancing the performance of the hexapod are immediate aims of the project. Additionally, this collaborative work is demonstrating how it may be possible for new, multi-organizational manufacturing enterprises to be assembled over an advanced network. Electronically linked partners are building experience in conducting and integrating remotely performed manufacturing activities. They are developing, for example, some of the prototype interfaces and other standards necessary to support geographically distributed design and manufacturing capabilities.

The Hexapod project represents how NIST, through the Information-Based Manufacturing Program, is anticipating and addressing the measurements and standards issues associated with leading-edge manufacturing technology of interest to U.S. industry. By improving the physical mechanics of how to work with this new class of machines this project is resulting in NIST and its industry, other government, and academic partners:

- developing and disseminating to industry an understanding of machine performance characteristics

- demonstrating interactive, remote experimentation capabilities

- providing input to national and international standards committees and users groups that are determining the best methodologies for evaluating and characterizing the performance of these machines

- developing a knowledge-base of machining experience

- improving machine metrology and control systems

Another example of the program's accomplishments relating to capabilities and competence in this area comes from a project that was initiated during the first program expansion involving cross-OU collaboration at NIST. This project, which is focused on automating construction sites, seeks to exploit advanced networking technologies to realize tremendous opportunities to trim construction time and reduce the need for costly rework. In this BFRL-led project, NIST and its partners are building the measurement and standards foundation so that

- the status of a construction site can be assessed instantaneously

- machinery can be operated remotely

- data flows seamlessly and can be easily integrated, providing workers and managers with the information they need, when they need it

To elaborate, geographically distributed project partners are developing the infrastructure for modeling, simulation, and automation of dangerous or error-pone construction tasks. This infrastructure will also be used for remote site-management capabilities and up-to-the-moment access to all site-related information, from architectural designs, to subcontractor schedules, to the status of machinery. Emphasis is on construction-site measurement capabilities-a necessary precursor 
for automation-and technologies for gathering, exchanging, and analyzing information. The project is also working with industry to develop wireless metrology and jobsite communications standards that will bring the IT revolution to the construction site.

Figure 3 below shows a portion of the physical testbed at NIST where research is occurring on this project. In the figure, a NIST researcher is shown controlling an automated, robotic, construction platform via a graphical interface. Among the results of this project are the development of general automation for construction sites, beyond the concepts of robot bulldozers; the development of wireless, job-site communications standards; and the development of new, cutting-edge field sensing technologies.

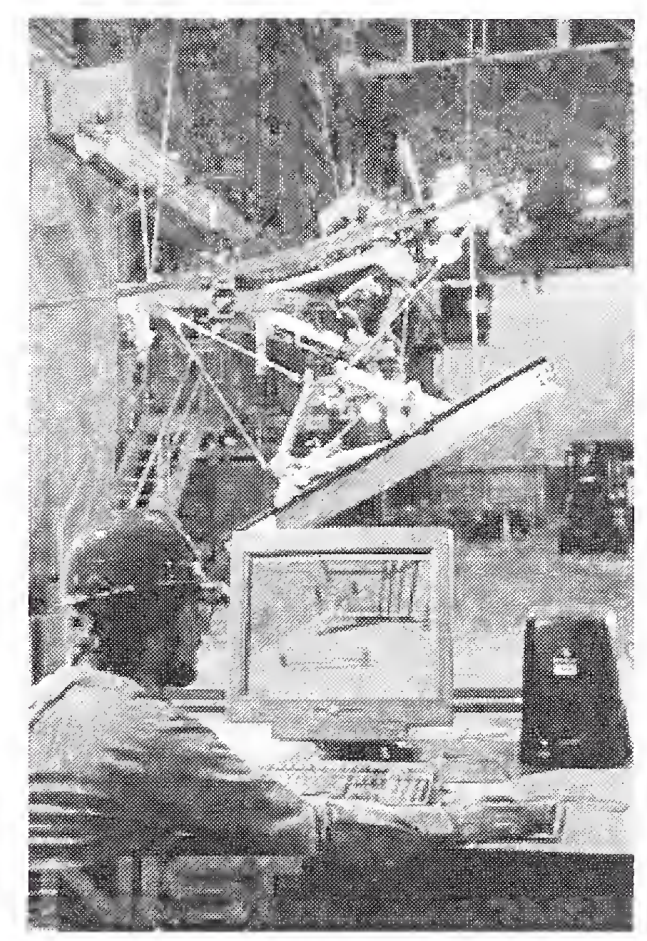

Figure 3. NIST Construction Automation Testbed

\section{The use of modeling and simulation to advance research}

This has been demonstrated by

- the development of high-fidelity, accurate, virtual machining operations by MEL

- the validation of manufacturing equipment motions, errors, and structural dynamics by MEL

- the provision of virtual testbeds that emulate the operations of complex building systems and operations by BFRL 
- the simulation of forming operations in virtual die design to prepare standard mechanical tests for sheet metal forming by MSEL

- the modeling of welding processes and sensor data to calculate weld quality in real-time by MEL and MSEL

With the power of today's computing technology, manufacturers are beginning to realize that processes, designs, and other applications can be developed efficiently when they are tested and proven out in the computer domain first, and then transitioned to the physical domain. This basic idea is summarized for manufacturing by, "First part correct."

These concepts can be captured in the phrase "virtual manufacturing." From visions of complex computer models of factories in operation, to machine tool models, to full immersion in virtual worlds, all of these concepts-and others as well-have come to mean virtual manufacturing. In the Information Based Manufacturing Program, virtual manufacturing has meant primarily the incorporation of computer-based modeling and simulation into manufacturing to improve how a particular process or operation is performed.

The hexapod project described previously in this report has incorporated virtual manufacturing concepts into its R\&D efforts. Figure 4 below depicts an example of a virtual manufacturing tool that has been used in this project.

Figure 4. Octahedral Hexapod Machine Ghost Image Simulation

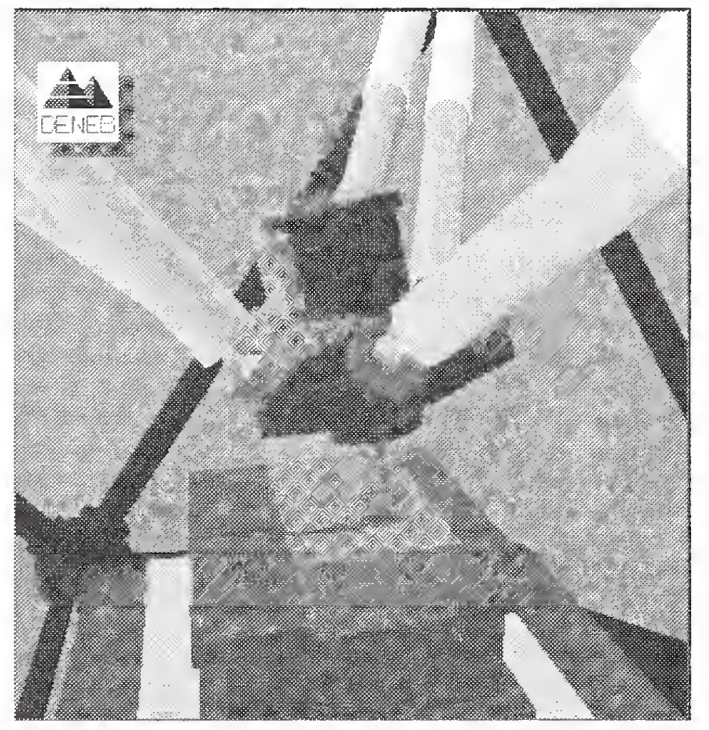

The software tool shown in Figure 4 is a "ghost image" simulation that is used to assist the performance characterization process for the NIST hexapod machine. This tool is valuable because the project is working to develop and document an understanding of the machine's performance characteristics to determine whether this new class of machine can become viable in a commercial sense. This sense of performance understanding must be developed through rigorous measurement 
and testing of the machines, and computer-based modeling of the machines in operation can greatly enhance the ability to do this.

The ghost image simulation tool uses dynamic graphics that incorporate measured machine performance parameters into a simulation of the machine in operation. Such a simulation shows how the machine operates for a particular process based upon actual performance characteristics that have been measured. These characteristics include, for example, geometry and backlash errors. This simulation is displayed simultaneously with a simulation of how the machine would perform ideally for the process if there were no machine errors, such as geometric inaccuracies. The simulation that depicts how the machine actually performs is displayed as a semi-transparent, ghost image that is projected on top of the simulation that depicts how an ideal machine should perform.

Tools such as this offer engineers the ability to quickly and accurately identify when errors occur, then focus on solving those machine performance characteristics that result in machining errors. These types of tools allow this to occur in a non-destructive environment where all proving out can be performed in the computer domain. The intent here is the development of a comprehensive visual model of manufacturing equipment operation that is based upon a rigorous understanding of the equipment's performance capabilities. This understanding of manufacturing capabilities can ultimately lead to the realization of product designs where the first part that is produced is correct.

Another active project that is exploring and exploiting virtual manufacturing concepts is the MEL-led Machine Tool Performance Models and Machine Data Repositories project. This project is also developing rigorous models of manufacturing equipment performance capabilities that are based upon actual, measured performance parameters. The models produced in this project incorporate representations of machine errors in a generic data structure. The NIST role in this project is primarily associated with the development of data structures that are, in fact, generic so that they can be applied to different classes of machine tools and manufacturing equipment.

The models represent a means by which an organization's shop floor manufacturing capabilities, or those of a supplier perhaps, can be accurately incorporated into design processes. This is an important concept in reducing manufacturing problems that can occur once a product or process design has been finalized.

Figure 5 below shows an example of a software tool that uses performance parameters for classes of machine tools to simulate parts produced on these machines. The simulation tool shown in the figure creates "whiskers" on a turned part to visually depict how spindle error on a lathe can result in a part with too much or too little material removed during a turning process. 
With tools such as this, engineers have the ability to create producible designs. Designs can be created with a high degree of certainty that they can actually be manufactured by allowing the designs to be validated and proven in the virtual sense based upon the measured performance characteristics of available manufacturing equipment. These tools also offer planners the ability to select certain pieces of equipment to match their capabilities to the specifications called for in product designs.

Figure 5. Simulation of Part Error Resulting from Turning Machine Geometry Error

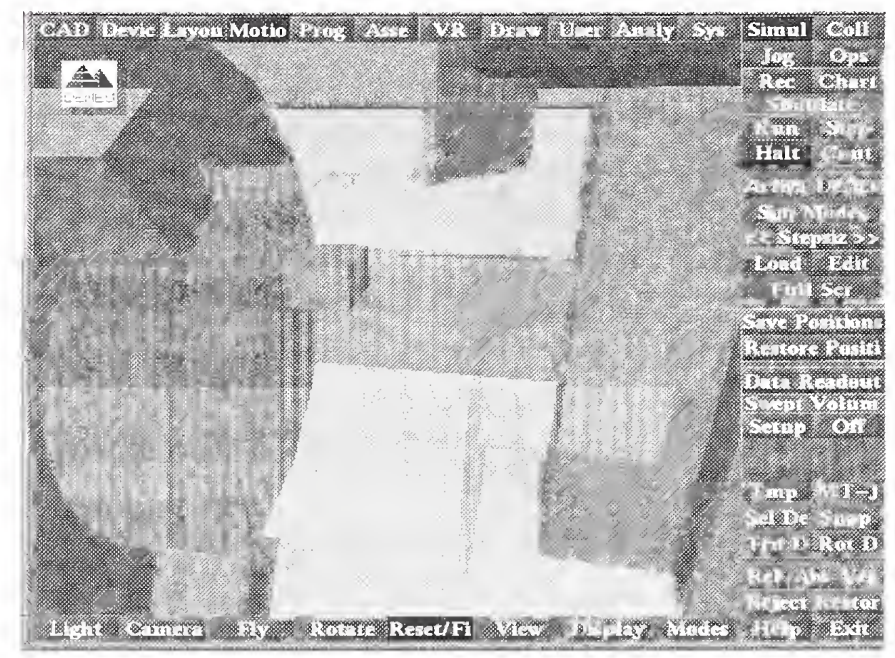

This document's Appendix provides additional information about how the projects within the Information Based Manufacturing Program address and leverage the concepts of virtual manufacturing.

\section{MEASURES OF PROGRAM SUCCESS}

In addition to the development and demonstration of the technical competencies described in the previous section, a number of other parameters exist by which the successes of the Information-Based Manufacturing Program can be measured. These parameters are described in this section to provide a more quantifiable means of representing and measuring program success.

\section{Fostering cross-OU collaboration throughout NIST}

The Information-Based Manufacturing Program has included 16 projects that have been technically led by each of the seven NIST Laboratories, plus the MEP Program. These projects, which have involved the participation of over 100 NIST staff members, have relied upon multi-disciplinary expertise that is resident across multiple NIST OUs to achieve goals and objectives. These collaborations have demonstrated how valuable the cross-fertilization of project teams with expertise 
from varied disciplines can be in the conduct of major, complex research projects. This, in turn, has also demonstrated a new sense of excitement across NIST in terms of what can be accomplished through multi-OU collaboration.

MEL has participated in all Program projects, and MEL's participation in cross-OU collaboration has existed in multiple forms, including:

- performance of research in pursuit of specific project objectives

- provision of IT support services to projects via the NAMT

- support and participation in technology demonstrations and focused technical workshops associated with specific projects

- provision of planning and other support services to projects, such as assistance with technical workshops and demonstrations

Without the Information-Based Manufacturing Program, these collaborations would very likely not have occurred and consequently, the successful projects and results described in this document would not have occurred either.

\section{Producing Traditional R\&D Program Outputs}

In addition to the other parameters mentioned in this section, the Information-Based Manufacturing Program has also produced traditional outputs that NIST uses to measure the success and impact of its various programs. These outputs include the following:

- publication of over 30 technical papers

- citation of the Program in 8 published news articles and periodicals

- execution of approximately 35 Cooperative Research and Development Agreements

- nearly \$2 million of investment from other government agencies into Program technical efforts

- the conduct of approximately 20 NIST-hosted technical workshops and symposia

- the delivery of Program presentations by Program staff at over two dozen technical conferences, workshops, and symposia, both domestic and international

\section{Fostering partnerships with customers of NIST}

Over 100 organizations from industry, other government agencies, and academia have worked in partnership with the projects of the Information-Based Manufacturing Program. Partnership on these projects has assumed a variety of forms, and several different vehicles have been used to define organizational partnerships that are both formal and informal. Means to enter into formal partnership arrangements include Cooperative Research and Development Agreements, the NIST Industry Fellows 
Program, and contracts. Intellectual property concerns, if any, and the expected outcome of joint efforts have usually dictated the type of arrangement. Informal partnerships have included standards committee and users group participation.

Project partners have used the NAMT to leverage and access resources in the conduct of collaborative R\&D. The means and extent to which resources are leveraged and accessed by project partners, especially those resources that are physically located at NIST, are determined by several factors. These factors include the nature of the work being performed, relevant technical issues, and relevant contractual stipulations that may be associated with a particular organization.

The following organizations are partners in various capacities on Information-Based Manufacturing Program projects. The lists below are not comprehensive, as only six organizations representing industry, government, and academia are listed here. These lists are provided to indicate the types of organizations that use the NAMT as collaborators on NAMT-supported projects. Additional information about partnerships associated with the NIST Information-Based Manufacturing Program can be found in [1].

$\underline{\text { Representative Industry Organizations }}$

- Caterpillar

- Boeing

- Honeywell / AMD

- Raytheon

- Ingersoll

- United Technologies / Pratt \& Whitney

Representative Government Organizations

- NASA Johnson Space Flight Center

- Department of Energy Argonne National Laboratories

- Department of Energy Sandia National Laboratories

- Department of Energy Oak Ridge National Laboratories

- Department of Energy Lawrence Livermore National Laboratories

- Department of Defense Manufacturing Technology Program

$\underline{\text { Representative Academic Organizations }}$

- University of Maryland

- University of Florida

- University of North Carolina-Charlotte

- Georgia Polytechnic Institute

- Carnegie Mellon University

- Ohio State University 


\section{Upgrading NIST networking capabilities}

Figure 6 below shows the topology of the network linkages established in the Information-Based Manufacturing Program through the NAMT. The figure shows the network connectivity for buildings and laboratories both around the NIST campus in Gaithersburg, along with how this connectivity is extended to non-NIST locations in the wide area.

Figure 6. Program Network Topology

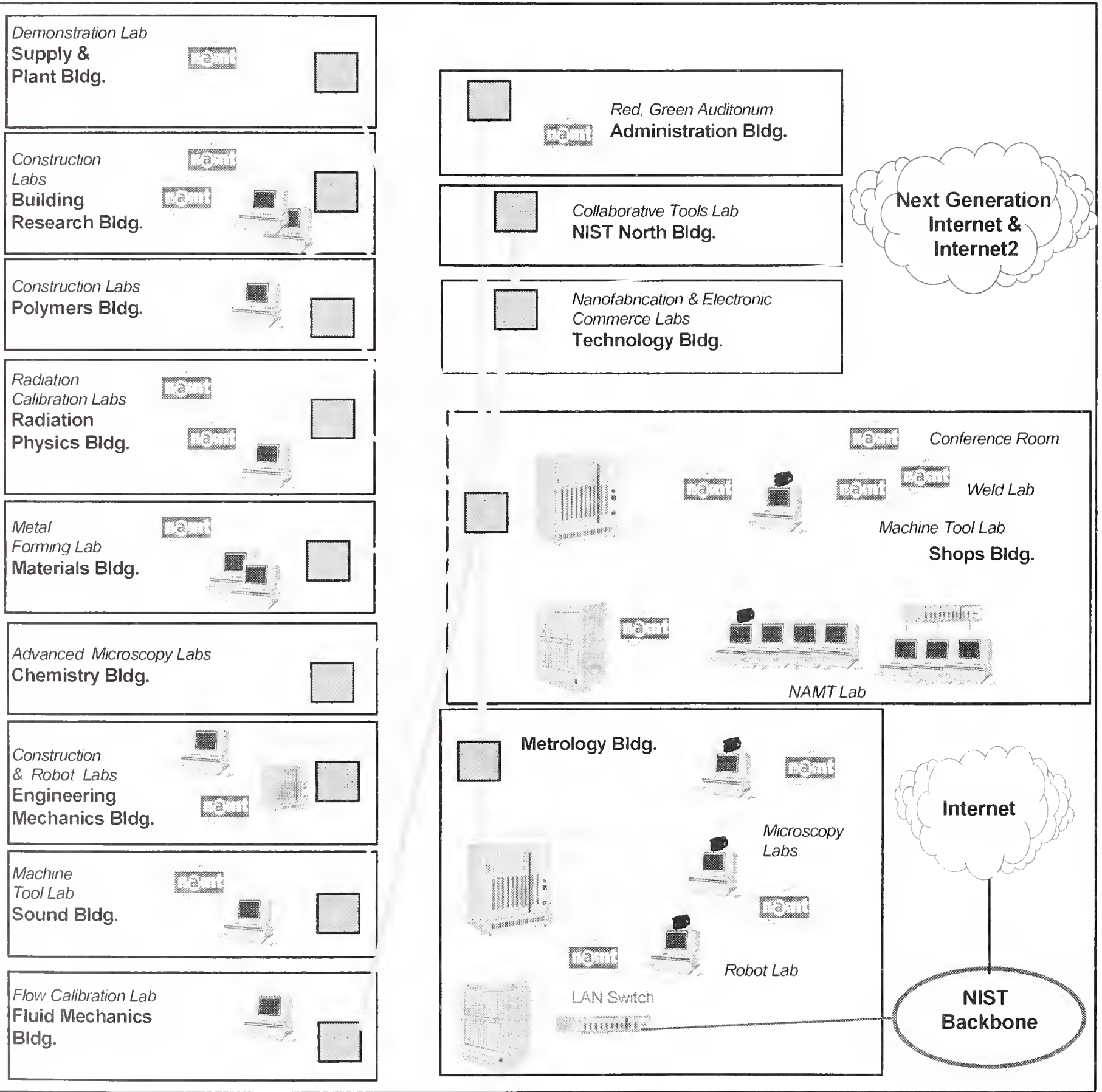


As detailed previously in the NAMT section of this document, the Information-Based Manufacturing Program has built a collaboratory on the NIST campus that is supported by the NAMT. This is a significant accomplishment because over a dozen buildings containing more than two dozen laboratory facilities across NIST have been linked together through high-performance network technologies. Prior to the existence of this Program and the NAMT, these high-performance linkages did not exist. As a result of this Program and the NAMT, scientists and engineers working in these linked facilities can perform collaborative research where the facilities are online in such a way that simultaneous access to voice, video, and data information allows for the quick and efficient leverage of geographically distributed resources. The technical focus areas of the NAMT Collaboratory-and also the focus areas of the Information-Based Manufacturing Program's projects-are depicted in Figure 7 below.

Figure 7. Program Technical Focus Areas
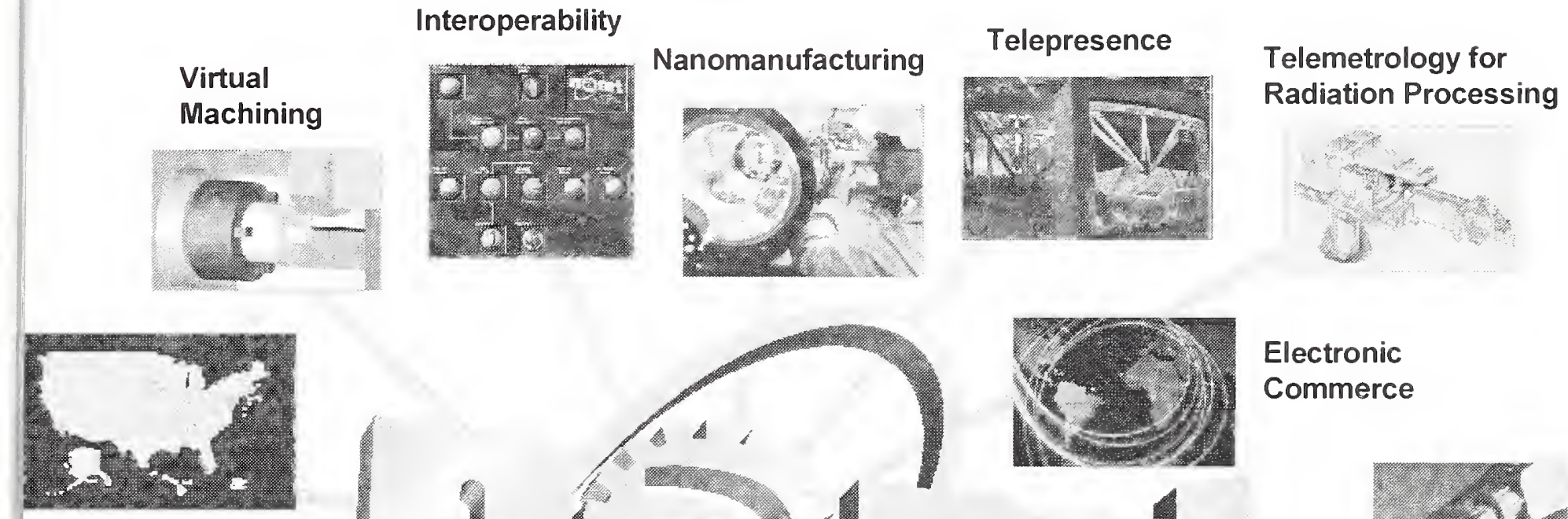

IT for Supply Chains

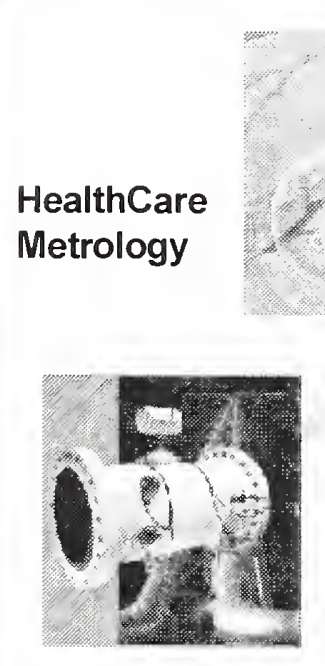

Telemetrology for Gas Flow

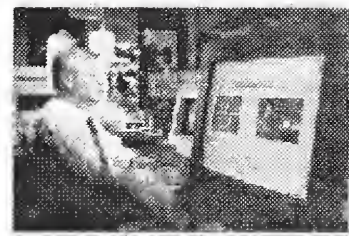

On-Line Length Calibrations

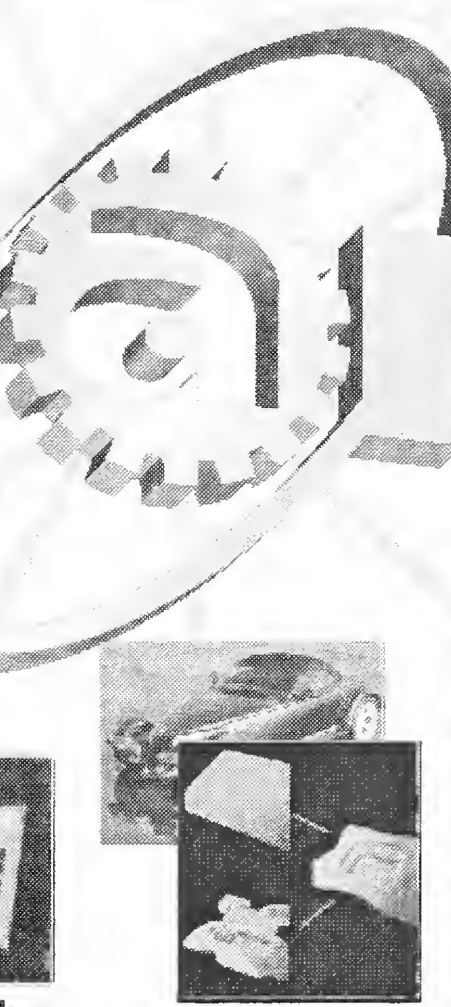

Die Design

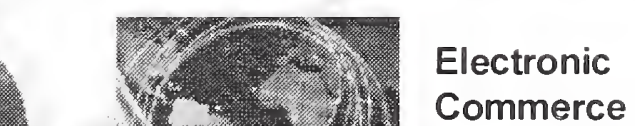

Commerce

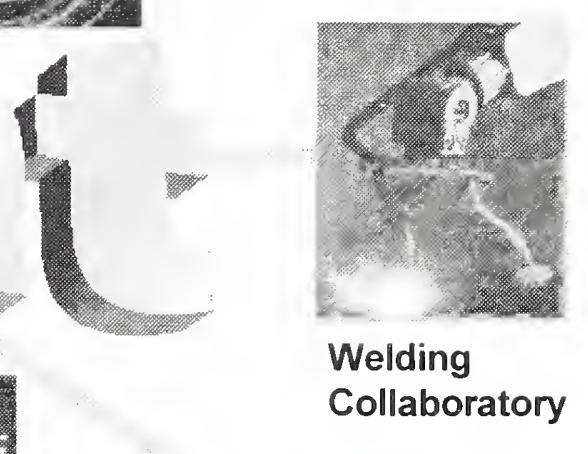

Automated Construction 
Additional information about the networking technologies and capabilities utilized in the Information-Based Manufacturing Program can be found in NISTIR 6383, "The National Advanced Manufacturing Testbed."[1]

\section{Demonstrating technical feasibility and leading-edge capabilities to NIST customers}

The Information-Based Manufacturing Program has provided over 150 technical demonstrations since 1996 for audiences representing industry, government, and academic organizations. The demonstrations provided by the Program have been a visible and tangible means by which leading-edge, technical concepts and results have been shown to be feasible to a wide cross-section of individuals and organizations representing existing and potential customers of NIST.

Demonstrations have used various levels of networking performance and capabilities. Several demonstrations have involved extensive reliance upon the NAMT ATM network backbone, while others have shown how simple, commodity Internet service can enable the conduct of collaborative research and measurement services.

Additional information about Program demonstrations that have involved the NAMT can be found in [1].

\section{SUMMARY}

The Information-Based Manufacturing Program has been developing competence across NIST in the application of IT to improve and advance the conduct of manufacturing research and the delivery of measurement and testing services. The Program, which has been in operation since 1996, is led by the NIST MEL and involves the participation of all of the NIST Laboratories, plus the NIST MEP and ATP Programs.

The Information-Based Manufacturing Program has created an increased sense of collaboration across NIST OUs, and it has played a significant role in demonstrating how IT can be used

- to better conduct research for and with NIST's customers

- to deliver NIST measurement and testing services more efficiently through on-line capabilities

- to put the right tools into the hands of the NIST researchers who need them, and do it at the right time

The NAMT has played a significant role as a physical testbed that supports the IT needs of the projects of the Information-Based Manufacturing Program. With the 
NAMT enabling various capabilities, the Program has focused on demonstrating feasibility of an array of different concepts, ranging from new ways for multiple organizations to partner with one another, to new technology, measurement, and standards development and applications. The Information-Based Manufacturing Program has been a key driver for demonstrating how NIST is embracing new capabilities and technologies in the conduct of its agency mission in the new millenium. 


\section{APPENDIX: $\quad$ PROJECT SUMMARIES}

PROJECT TITLE:

LEAD NIST OU:

YEAR ESTABLISHED:

\author{
Nanomanufacturing of Atom-Based Standards \\ Manufacturing Engineering Laboratory \\ 1996
}

\section{$\underline{\text { Overview }}$}

There are two major industry trends that are driving forces for this project. First is the ever decreasing dimensions and tolerances posed by the semiconductor and datastorage industries. Industry projects that within a decade the critical dimensions of microelectronic devices will be near 100 nanometers, requiring dimensional standards with uncertainties near 1 nanometer and a degree of geometric perfection near to that of an ideal atomic lattice.

The second major trend in industry is the demand (in major part produced by the first trend) for ever cleaner and highly controlled manufacturing environments. Currently these trends are being manifested in the move from clean rooms to minienvironments to clean machines and the use of standard mechanical interface pods to transfer materials between the manufacturing tools. As a result NIST is undertaking development of atom-based dimensional artifact standards, where feature size and geometry derive directly from that of atomic lattice. Some such artifact standards are being fabricated, transported, and used at different sites, spending their lives under vacuum. At the same time, microelectronic devices themselves are being produced at different manufacturing sites with expensive, specialized equipment carrying out different steps of the overall manufacturing process. These steps include research and development, design, fabrication, inspection, processing and repair. Much of this is dependent upon sophisticated computer modeling, communications and control.

\section{Goals}

The goals of this project are

- to support the development and deployment of the technology of distributed fabrication and use of nanometer-scale dimensional artifacts

- to use computer modeling and simulation for the development of mechanical systems and components, including the artifact transport system

- to develop and demonstrate remote teleoperation of scanned probe microscopes

- to incorporate and deploy links by advanced computers and communications for high-speed video, voice and data transmission among collaborators

\section{Benefits}

Benefits from the project are 
1. next generation calibration standards with accuracy meeting industry's projected needs

2. interface standards for artifact transport systems (see description below)

3. interface standards for remote teleoperation of scanned probe microscopes

There are three major task areas.

\section{Atom-Based Dimensional Calibration Standards}

The first major task of this project has been the demonstration of feasibility of fabrication of artifacts with dimensioned features derived from atomic-lattice properties. The size and tolerances of dimensioned features in micro-, nano-, and quantum-electronic devices are rapidly decreasing. This creates a need for artifacts as calibration standards that have feature sizes and uniformities in geometry that are nanometer and sub-nanometer in scale. Rather than depending on the removal or addition of material to form dimensioned features of arbitrary size and shape, an alternative for the fabrication of calibration standards with nanometer-scale dimensions and high geometric perfection is to use controlled natural processes (such as lattice dislocations). These processes yield features of size and shape determined by crystalline lattice spacings and geometries. In collaboration with vendors and users of commercial dimensional calibration standards, the project is demonstrating feasibility of fabricating such atom-based artifact standards of use to the U.S. microelectronics industry.

\section{Artifact Transport System}

The second major task has been the development of a portable, shippable chamber with a standard mechanical interface. Such a chamber will allow physical transport under vacuum of wafers and other substrates undergoing processing in high-vacuum systems in clean-room facilities at geographically different locations. These different locations can be different facilities at the same site or different sites. The shippable chamber, called a "vacuum suitcase", is an essential element in the move from a physically monolithic processing chamber to a physically distributed processing system. In collaboration with vendors and users of vacuum equipment, this project has developed a prototype "vacuum suitcase." This "vacuum suitcase" is for use in the transfer of wafers and substrates between the NIST Ultra High Vacuum Scanning Tunneling Microscope, the NIST Molecular Beam Epitaxy machine, wafer-processing systems and related industry systems.

\section{Teleoperation of Scanned Probe Microscopes}

The third and last major task has been the demonstration of remote diagnostic operation of scanned-probe-microscopy systems using standard data representations. There is an increasing requirement for microelectronic wafer processing and device fabrication completely within clean-room environments. This 
has created a growing need for not only automated control of processes and roboticmanipulation of wafers and carriers, but remote "through-the-clean-room-wall" teleoperation of scanned probe microscopes used for inspection of fabricated micro structures. A need also exists for remote "from-a-vendor's-firm" teleoperation of such microscopes for instrument trouble-shooting. In collaboration with vendors and users of scanned probe microscope instruments, the project has developed a test-bed for the identification of standard interface needs and issues in scanned probe microscope teleoperation. 
PROJECT TITLE:

LEAD NIST OU: YEAR ESTABLISHED:
Machine Tool Performance Models and Machine Data Repositories Manufacturing Engineering Laboratory 1996

\section{Overview}

To reduce costs and respond rapidly to changing customer needs, large companies are relying increasingly on a network of suppliers and outsourcing a significant percentage of their manufacturing needs. This type of geographically and organizationally distributed manufacturing requires better communication and improved coordination and utilization of internal and external manufacturing resources by all the participants.

Because of the need to shorten design and production cycles, designers and production engineers must collaborate closely to determine the optimum use of resources required to turn designs into real products, without extensive prototyping. Prototyping is usually an iterative process, taking considerable time and effort before actual production can begin. Creating a virtual manufacturing environment to simulate the complete manufacturing cycle and carry out this iterative process in the virtual domain presents a unique opportunity for industry to reduce time for new product introduction.

\section{Goals}

The goal of this project is the development of engineering tools that enable design and manufacturing engineers to predict machine tool performance and ensure that parts can be machined to specification with a minimum of prototyping. These engineering tools include data structures and low-order machine models that represent actual machine behavior. They also include mathematical representation of actual part geometry, including dimension and form errors. Additionally, virtual machining algorithms, virtual inspection algorithms, standardized data formats, and remotely accessible machine data repositories are being developed.

The project has sought to replace actual machining and inspection of parts during prototyping with virtual machining and virtual inspection modules incorporated into a CAD/CAM system. The virtual machining module simulates the movement of the culting tool when making a part. In this simulation, the effects of error motions, predicted from machine-tool characterization data are reflected in the tool path. Virtual machining results in an electronic approximation of the part that can be inspected by the virtual inspection module. The virtual inspection module determines the uncertainties associated with the selected inspection plans and equipment. These uncertainties can be checked against the specified design tolerances of the part. This virtual environment makes it possible to optimize the manufacturing process by trying different machines and making changes to the process plans or part designs. 
One major challenge has been the development of a data structure for electronic representation of machine performance and error characteristics. Such a data structure can enable different manufacturing entities to effectively share information on available manufacturing equipment. The types of information necessary for this purpose include error characteristics of machine components, descriptions of machine structure, axis designations, error notations and sign conventions. Based upon the developed data structure, a data repository for storing machine performance information is being created. This repository is being populated by major manufacturers and their suppliers, as well as machine tool vendors. Algorithms are being developed to access performance data from this repository and to apply the data in predictive machine models.

\section{Benefits}

This project is contributing to a variety of important benefits, including:

- optimum and speedy allocation of in-house and/or supplier manufacturing resources, which can significantly shorten time for new product introductions

- improved quantitative information exchange between design engineers, manufacturing engineers and process planners

- optimization and quality assurance of existing manufacturing processes 
PROJECT TITLE:

LEAD NIST OU: YEAR ESTABLISHED:
Characterization, Remote Access, and Simulation of Hexapod Machines Manufacturing Engineering Laboratory 1996

\section{Overview}

The rapid production of quality parts with complex contoured geometric features requires machine tools that combine speed, accuracy, stiffness, and multiaxis versatility. In addition, manufacturers look for qualities such as ease of installation and portability to enable plants to be reconfigured to meet changing market demands. A new class of parallel-actuated machine tools based on the Stewart platform mechanism presents new possibilities to meet these needs. However, much remains to be learned about the characteristics of these 'hexapod' machine tools before they can see widespread production application.

Industry workshops have highlighted the need for:

- an in-depth understanding of the characteristics of these new machines

- standard test methods and measurement procedures to evaluate their performance

- a reservoir of application experience from which to draw

- modeling and simulation tools for developing applications and test methods

- remote access capabilities to make it easier for external collaborators to interact and participate in the work being done

- examination of controller and integration issues

\section{$\underline{\text { Goals }}$}

The goal of the project has been the development of methods to characterize and extend the limits of performance of a new class of Stewart platform-based machine tools in terms of accuracy, productivity, and versatility through the development and implementation of virtual and distributed manufacturing technologies.

The NAMT Hexapod project team, in collaboration with external partners, has been performing work in the three areas of characterization, remote access, and simulation to address the needs discussed above. The work at NIST is performed using the Octahedral Hexapod machine, which was installed at the Gaithersburg, MD site in May, 1995. To the extent possible, models, measurement techniques, and other project results are developed in a generic form to allow them to be easily applied to other machines with a parallel arrangement of actuators. NIST researchers are participating in a national Hexapod Users Group to coordinate research activities and share results. 
Benefits

Potential benefits of Hexapod machines cited in the trade literature include increased stiffness, higher accuracy, higher speed and acceleration due to reduced moving mass, and reduced production and installation costs. This project's results include:

- test procedures and standards to assess, describe, and compare the performance of these new machines

- opportunities for machine tool users to gain first-hand experience with this new technology

- networked sensor and control information for remote experimentation, interaction, and integration

- program validation, workspace analysis, part placement, and other simulation tools for faster application development

- performance enhancements, such as calibration techniques and feedback metrology systems, for higher accuracy

This project has been working to produce benefits in the technical areas as detailed below.

\section{Characterization}

The approach used to develop performance evaluation techniques has included the ANSI/ASME B5.54-1992 Methods for Performance Evaluation of Computer Numerically Controlled Machining Centers [4] and ISO 230 [5] as starting points. Modified versions of these test procedures are being proposed and tested for hexapod machines. Performance enhancements, such as improved calibration and feedback metrology techniques, are also being used as an improved understanding of machine behavior is gained.

Remote Access

For remote interaction, high-speed communications links, such as ATM networks, have been used to provide real-time transfer of audio/video and sensor data to researchers at remote sites. An Internet-based version of similar capabilities has also been developed that provides services at a lower cost, but with some compromise in performance.

To examine controller and integration issues, an open architecture controller is being installed and tested on the Hexapod. This controller is based on Application Programming Interfaces and software developed under the NIST Enhanced Machine Controller Program [6]. 


\section{Simulation}

From the validation of machine motions to detailed investigation of the errors and structural dynamics of the Hexapod, a comprehensive set of modeling and simulation tools is needed. These simulation capabilities are being developed in an incremental fashion, gradually adding to the fidelity and detail of the modeled behavior. This is reducing the risks and increasing the effectiveness of the hexapod research efforts. 
PROJECT TITLE:

LEAD NIST OU:

YEAR ESTABLISHED:
Framework for Discrete Parts Manufacturing Manufacturing Engineering Laboratory

1996

\section{Overview}

Driven to improve competitive performance, U.S. manufacturers invest billions of dollars each year in information technology. A substantial portion of this investment is allocated for creating and integrating applications software. Chief among these integrated applications are manufacturing execution systems (MES), product data management (PDM) systems, database management systems (DBMS), computer aided manufacturing (CAM), and factory control systems, as well as planning and scheduling systems. Many of these applications and most of the software used in their integration are non-standard, one-of-a-kind creations. Now, manufacturers are trying to replace their in-house software systems with standards-based, off-the-shelf products. They want to reduce their outlay of capital and human resources on systems development, integration, and maintenance in order to focus on core manufacturing competencies and to improve operational flexibility.

Industry requires the capability to rapidly integrate best-in-class applications, improve interoperability, defer obsolescence, increase system stability, and integrate widely distributed manufacturing operations. Information technology standards provide these benefits, while reducing technical and financial risks. However, no single standard exists that will satisfy all of the system interoperability needs of manufacturing organizations. Consequently, development and adoption of suites of information technology standards are key elements in the competitive strategies of many U.S. manufacturing organizations. Validation testing of candidate standards is a critical technical ingredient of these strategies. Manufacturers use validation testing to determine profiles of standards. Information technology vendors use test results to garner marketplace confidence and to reduce technical risks associated with the adoption of new technologies embedded into modern, complex manufacturing systems.

\section{$\underline{\text { Goals }}$}

The Framework Project is providing industry with tests and methods for analyzing and validating emerging manufacturing information standards and technologies. Work has been focused on information models for manufacturing, software application interface definitions, object-oriented class hierarchies, data access protocols, scheduling and control strategies, integration mechanisms, and communication architectures.

This project is performing analyses and validation testing of standards, pre-standard specifications, and other so-called middleware technologies developed by industry consortia. The approach taken in this project has been to implement a distributed manufacturing software system based on the combined use of emerging standards 
and technologies. This test system consists of MES, PDM systems, DBMS, CAM systems, and factory control systems, as well as planning and scheduling systems.

Testing has been conducted against manufacturing scenarios representative of the defense, aerospace, and automobile industries and entailing the design, production, and inspection of discrete metal parts. The results of the implementation and testing form the basis for documented reviews of the specifications. These reviews address the internal consistency of each specification as well as the technical relationships between the specifications. These reviews are used as input to ongoing development within the various consortia and they are used in the formal consensus building process within standards organizations.

Benefits

Project benefits include:

1. Test-generated information that can be used in standards profiles, aiding strategic standards management.

2. Validation of emerging standards and technologies that can reduce the standards development cycle-time.

3. Standards-based information technology that can increase product quality, decrease time-to-market, reduce product development costs, and increase the efficiency of manufacturing operations.

4. The adoption and use of standards that reduce costs for manufacturing-system end users and allow system providers to profit from economies of scale. 


\section{PROJECT TITLE: Interface and Data Standards for Real-Time Construction Site Metrology \\ LEAD NIST OU: Building and Fire Research Laboratory \\ YEAR ESTABLISHED: 1997}

\section{Overview}

U.S. construction industries account for approximately $13 \%$ of the United States' gross domestic product [7]. Time is the primary issue in these industries, and even small construction technology improvements that enable the ability to finish a job correctly the first time and on-time offer the potential for major economic impact to this very significant industry. Real-time, wireless metrology capabilities form the foundation for industry's abilities to automate and improve operations at construction sites. This project has been working with industry to speed the development and application of the standards required to implement real-time metrology into automated construction site applications.

$\underline{\text { Goals }}$

The project has established a National Construction Automation Testbed for collaborative development with industry of interface data standards for real-time construction site metrology. This remotely accessible testbed facility incorporates site simulation tools for the planning and programming the operations of semi-automated machinery into the development of the interface data standards that are the primary focus of the project. The testbed is conducting construction experiments at full-scale to determine problem areas for information transfer.

\section{Benefits}

The project has been conducting research focused on the development and testing of non-intrusive site scanning, discrete component identification, three-dimensional dynamic databases, and multi-robot coordination. As a result of this research, project benefits include:

- metrology relevant to construction sites and processes, specifically in terms of open standards for real-time ("live") data exchange, inter-operability, and new, cutting-edge field-sensing technology

- Automation tools for on-site productivity, quality, and safety, where automation is not just "robot bulldozers," but includes data at your fingertips (measurements, CAD data, phone numbers), user feedback (3-D graphics, head-up displays), and derivative quantities and automatic object recognition 
PROJECT TITLE: LEAD NIST OU: YEAR ESTABLISHED:
Telepresence Microscopy and Microanalysis Chemical Science and Technology Laboratory 1997

\section{Overview}

Telepresence microscopy and microanalysis is the remote sharing and/or operation of microscopes/microprobes. The chemical microstructure of material specimens is critical to materials science and technology, semiconductor device development, quality measurements and process control, and failure analysis and evaluation. NIST microanalysis customers need the following:

- quick access to advanced microscopy and microanalysis instrumentation and expertise, which is especially critical to small start-up firms

- quality measurements of nanoscale dimensions and material chemistry properties

- quick time response for analysis that is demanded by process control

\section{Goals}

This project has established and is demonstrating remote communications with microanalysis customers from NIST labs via inexpensive teleconferencing hardware/software, as well as via more high-performance networking technologies. Low-end, communications-based systems utilize World Wide Web-based technology, specifically allowing for "over-the-shoulder" remote monitoring of NIST analysis experiments. This specifically targets small enterprises that account for a large percentage of the industrial community working in these areas. The high-end portion of the project is also developing, testing, and implementing the hardware and software that is necessary for remote control of these types of leading edge instrumentation at NIST labs. This specifically utilizes high bandwidth networking technology capabilities. This project is gaining critical research experience in telepresence operation by solving actual problems occurring daily within industry.

\section{Benefits}

Through demonstrating telepresence in the areas of advanced microscopy and microanalysis, this project has been enabling and demonstrating the possibility for and benefits of more efficient use of expensive, scarce resources and critical personnel at industrial central laboratories. The project has done this by providing remote, instantaneous, around-the-clock access to critical production facilities; by providing "just-in-time" analysis; and by improving communications within the service analysis industry.

Ultimately, standards and standard data will be created for telepresence microscopy and microanalysis. 


\section{PROJECT TITLE:}

LEAD NIST OU:

YEAR ESTABLISHED:
Virtual Die Design for Information-Based Metal Forming

Materials Science and Engineering Laboratory 1997

\section{Overview}

The metal forming industry is a several hundred billion-dollar industry in the United States, with a large portion being accounted for by the automotive industry. The development of new dies for stamped parts for a car model can cost several hundred million dollars, and typical time to production for a new car model of around 50 weeks. The time for producing and trying dies can be on the order of 25-28 weeks. Die fabrication costs tend to be so high because commonly used die design processes are typically sequential operations involving little concurrent communication among the various technical functions involved in the process [8].

\section{$\underline{\text { Goals }}$}

The project has been incorporating improved materials models into common finite element analysis packages to improve the constitutive laws associated with simulations of forming operations that are used in virtual die design. The project has concentrated on:

- the development of standard mechanical tests relevant to sheet metal forming, including plane strain tensile tests and channel forming tests

- the development of accurate simulations of material responses in forming processes

- on-line techniques for remote data acquisition and control of forming operations.

\section{Benefits}

Capabilities enabled by virtual die design include more rapid fabrication of sheet metal forming dies and higher precision stamped parts with tighter dimensional tolerances. Improved intercommunication between the producer, supplier, and designer of dies is also a benefit. Ultimately, the capabilities developed in the project demonstrate the capabilities to implement die design and fabrication scenarios where the number of tryouts are reduced to one - where the first die that is designed and fabricated is the correct one. 
PROJECT TITLE:

LEAD NIST OU: YEAR ESTABLISHED:
Design, Manufacture, and Calibration of Radioactive Seeds and Other Radiation Sources

Physics Laboratory

1997

\section{Overview}

Application of radiation sources for interstitial cancer therapy (presently 50,000 patients per year in the US) and for cardiovascular therapy to prevent restenosis following angioplasty (potentially 400,000 patients per year in the US) is extremely important for US health care [9]. Using the capabilities of the NAMT infrastructure, combined with robotics and communications software development from the Intelligent Systems Division, the lonizing Radiation Division is developing and has demonstrated an interactive network for a hierarchical measurement system as a model for other national standards laboratories.

\section{$\underline{\text { Goals }}$}

The project involves a number of components in design, manufacturing, and calibration. The modeling and simulation methods, developed at NIST for determination of the radiation fields emerging from these small sources and the spatial distribution of dose absorbed in a medium, are regarded as benchmark quality by the international community of source users and manufacturers. These methods have been incorporated into many available computational codes, but the use of such complex codes can require significant training and intellectual investment. The project is making these calculations available for remote use through the World Wide Web. Additional effort is developing the necessary software interfaces and expert systems to allow source manufacturers and other interested parties to input mechanical-drawing information and other pertinent data to simulate the dose rate delivered by a potential source and to vary parameters to optimize source design.

The ability to accurately connect information on the incorporated radioactivity and the dose rate delivered to a medium depends critically on knowledge of the absolute activity of the radionuclide present in the source. NIST is a world leader in radionuclide metrology and works with manufacturers to provide factors for commercially available calibrators of radioactive sources. This program includes many new sources being considered for brachytherapy, with the information made available to manufacturers on the world-wide web.

Modeling and simulation technologies are being used in the design of hazardous radioactive sources. The effect is the reduction of radiation doses to manufacturing personnel, allowing the ability to test "virtual designs" of new radiation sources, rather than building and testing each one.

In subsequent calibrations the sources must be handled several times at different institutes. An improved communications network that links manufacturers, NIST and 
calibration laboratories is improving communication and product quality while simultaneously reducing source handling and the number of calibrations required.

Benefits

Manufacturing and sales of these radioactive sources represent one of the fastest growing segments of the US economy. ABC Evening News reported in 1997 the successful use of I125 in prostate cancer therapy, and the manufacturer of these sources has reported sales increasing at $10 \%$ per month. The Wall Street Journal reported on the new application of seeds to prevent restenosis following balloon angioplasty, and all of the source manufacturers, major medical centers, and instrument makers are exploring new approaches through research teams.

This project is impacting and enhancing all these efforts, and NIST is developing a new paradigm for how these sources are designed, manufactured and calibrated. Project approaches to networked source handling and calibrations can be quickly introduced to the calibration community at the major medical calibration centers M.D. Anderson Cancer Center, Memorial Sloan Kettering Cancer Center and University of Wisconsin. The manufacturers also have immediate benefits in the form of lower design and construction costs from robotics systems designed by NIST. 


\section{PROJECT TITLE: $\quad$ Internet Commerce for Manufacturing LEAD NIST OU: YEAR ESTABLISHED: \\ Electronics and Electrical Engineering Laboratory 1997}

\section{Overview}

The Internet Commerce for Manufacturing project (ICM) was established to work with industry to enable both small and large organizations to make effective use of Electronic Commerce (EC) technology across a portion of the printed circuit assembly (PCA) supply chain.

\section{Goals and Benefits}

The project is validating and demonstrating the use of open systems and standards to efficiently share both technical and business data across organizations. The project is also aiding the development and testing of standards identified as critical.

The ICM project is

- Gathering and documenting industry requirements and current practice through a series of Scenario Development/Strategy Workshops. This information is used to develop an Activity Model to guide the project, and to publish a Business Case to document the economic motivation for migrating towards Internet commerce in the PCA industry. The Business Case highlights the industry's structural changes, as well as suggesting an appropriate industry and government response to facilitate the adoption of EC standards and technologies.

- Identifying and developing needed standards. A Standards Roadmap from the project reflects the availability and status of standards for the exchange of both technical and business information. The ICM team is participating in the development and testing of those standards from the Standards Roadmap identified as critical to the migration towards Internet commerce, such as the IPC GENCAM standard.

- Developing an Internet Commerce for Manufacturing testbed at NIST. This testbed validates the ability to use suites of standards identified in the Standards Roadmap to address the industry needs identified in the Business Case document. Industry and academic partners serve as demonstration nodes within the scenario, with NIST developing enabling tools for demonstration purposes and to facilitate further development.

- Disseminating results to industry, academia and other agencies through participation in a variety of in-house and conference demonstrations; publication of the business case, standards roadmap, and proceedings from all workshops; working with the NIST Verification of IT Solutions for SMEs (VITSS) project in the transfer of research results to industry via national recommendations to Manufacturing Extension Partnership Centers and actual product offerings (not to be developed by NIST). 
PROJECT TITLE:

LEAD NIST OU:

YEAR ESTABLISHED:
Virtual Cybernetic Building Testbed Building and Fire Research Laboratory 1998

\section{Overview}

Industry is experiencing increasing pressure to integrate more and more building control systems and services. This pressure creates a need for the ability to test and evaluate the complex interactions that are likely to occur under normal and adverse (e.g., ernergency) operating situations. Control manufacturers and service providers also need assistance in the development, testing, and certification of new products for building control and services. These tasks simply can not be accomplished using real buildings because of the complexity of the systems involved and because of need to maintain a comfortable and safe occupant environment in buildings at all times. They can only be done through simulation/emulation and the establishment of a Cybernetic Building Testbed where manufacturers can (1) bring the actual control products that they have under development, (2) obtain assistance in testing and evaluating their performance, and (3) perform interoperability tests with other manufacturers.

Researchers and service providers can use the Testbed either onsite or remotely to study system interactions, evaluate control algorithms, test the performance of new services, and develop national and international standards for integrated systems that will assure life safety, reliability, proper operation, and enhanced building system performance.

\section{Goals}

This project is creating a real time, distributed Cybernetic Building System Emulator called a Virtual Cybernetic Building Testbed (VCBT). It consists of a variety of simulation models (or emulators) that together emulate the performance of a typical cybernetic building system. The simulation models are interfaced with real state-ofthe-art and prototype control systems using the Building Automation and Control Network (BACnet) data communication protocol to provide a hybrid software/hardware testbed. This testbed can be used by NIST researchers, control manufacturers, service companies, and software developers to develop and evaluate control strategies and control products that use the BACnet protocol [10].

The VCBT emulates the performance of both fault-free and fault-containing heating and cooling equipment, different heating, ventilating and air conditioning (HVAC) systems, and the building shell, as well as life-safety, lighting, security, and vertical transport systems, and other services. Included are an advanced graphical user interface and remote accessibility to the VCBT through various communication interfaces such as telephone, the Internet, and the World Wide Web. 
This project combines the NIST BFRL's extensive experience with the modeling and simulation of buildings, HVAC systems, controls, and fires with the NIST MEL's expertise in systems integration, object oriented programming, the use of a Common Object Broker Architecture (CORBA), advanced information models and data bases, and the Virtual Reality Modeling Language (VRML). The project incorporates the NAMT high-performance network to exchange information among the various VCBT components in real time, allowing for both on-site and remote use of the VCBT by NIST customers.

Phase I of the project involved the development of an HVAC emulator to simulate the performance of a Variable Air Volume (VAV) air handling unit, three VAV boxes and three building zones using BFRL's HVACSIM+ program. Phase II developed a building shell emulator, a fire emulator, a more complex HVAC emulator, a building/HVAC Product Model, and a VRML based interactive VCBT display. The various VCBT components, distributed about different NIST locations, use the CORBA paradigm to provide a real-time, distributed emulation environment based on message passing between objects and client-server programming. Phases III and IV, which represent future activities, will involve the enhancement of the VCBT front-end and the expansion of the VCBT to include the emulation of additional building services, fault containing systems, and other services likely to be provided by outside service companies.

\section{Benefits}

The Virtual Cybernetic Building Testbed (VCBT) is a tool for researchers at NIST and other organizations to study the complex interactions that occur as a result of integrating different building services and systems. Of particular interest is the impact of integrating fire detection, smoke control, transportation, HVAC, and energy management systems on life safety. The availability of the VCBT as a testbed for testing and evaluating changes to the BACnet standard facilitates the extension of the BACnet protocol to cover lighting, fire detection, transportation, and other services.

Manufacturers of building controls and future cybernetic building products can use the VCBT to:

- develop and test algorithms

- evaluate the performance of new products

- perform interoperability testing with other manufacturers.

The existence and use of the VCBT by newly developing service companies can greatly speed up the development of new building services, such as fault detection and diagnosis, automated commissioning, building system optimization, and predictive maintenance. In addition, by providing both on-site and remote access to the VCBT, NIST has provided greater customer access to the research facilities of the Building and Fire Research Laboratory. 
PROJECT TITLE:

LEAD NIST OU:

YEAR ESTABLISHED:
Tele-Calibration of Gas Flow Meters

Chemical Science and Technology Laboratory

1998

\section{Overview}

Every year in the U.S. gas flow meters are used to measure trillions of dollars in goods destined for consumption here and abroad. For example, Americans use natural gas at the rate of $\$ 2.2$ billion per day and this gas is metered some five to seven times between the well head and the consuming burners. Gas flow meters are also extensively used throughout industry as process control monitors in applications that range from the measurement of power plant emissions to the control of oxygen flow for premature children in intensive care units.

In conjunction with the establishment and maintenance of the national measurement infrastructure that can assure the quality of these important measurements, NIST provides gas flow measurement standards and meter calibration services to US industry, other government agencies and academia. These services are provided for a gamut of gases, for widely ranging conditions for flows from $\left(1 \times 10^{4}\right.$ to $\left.8.5 \times 10^{4}\right)$ standard liters per minute $(\mathrm{slm})$ (or, $\left(1.67 \times 10^{2}\right.$ to $\left.1.42 \times 10^{3}\right)$ liters per second).[11]

Although NIST has provided calibration services in flow for approximately five decades, U.S. industry has critical needs for gas measurement traceability at much larger flow rates (ranging to approximately $1 \times 10^{8} \mathrm{slm}$ ) (or, to approximately $1.67 \times$ $10^{6}$ liters per second), at a wide range of working pressures (from (1 to 60) atm), and for a large number of gas species. The construction of facilities capable of handling such large flow rates at NIST is all but impossible due to capital and space constraints.

A small number of secondary metrology laboratories in the US, however, are capable of calibrating sensors at flow rates larger than those attainable by NIST. One such facility is the Colorado Engineering Experiment Station Inc. (CEESI), in Nunn CO, which provides calibrations at flow rates as large as $2.7 \times 10^{5} \mathrm{sIm}$ (or, $4.5 \times 10^{3}$ liters per second), with plans for a CEESI facility in lowa capable of flowing as much as 4.3 $\times 10^{7} \mathrm{~s} / \mathrm{m}$ (or, $7.17 \times 10^{5}$ liters per second) - the largest facility of its kind in the world.

\section{Goals}

This project has been using the NAMT information infrastructure to expand the range of NIST gas flow calibrations by making the facilities at CEESI available for use in NIST calibration operations over a wide-area, high-performance, computing and communications network. By installing NIST instrumentation and a NIST portable primary standard at CEESI's "Primary A" facility in Nunn, CO, this instrumentation can be accessed using Web-based technology by NIST personnel to provide NISTcertified calibrations outside NIST's Gaithersburg campus. 


\section{Benefits}

This project is benefiting U.S. industry by addressing the following needs:

- demonstration of the feasibility of using information technology to expand NIST's calibration services via tele-presence in U.S. secondary calibration laboratories.

- expansion of NIST's gas flow calibration capabilities by allowing NIST to calibrate meters in flows ranging from 3 times to possibly 500 times larger than those used prior to the establishment of the project

- implementation for the first time of CSTL's portable primary standard concept that promises to be a driving force for future practice in flow meter calibrations. 
PROJECT TITLE:

LEAD NIST OU:

YEAR ESTABLISHED:
Remote Calibration and Verification of Electrical

Standards

Electronics and Electrical Engineering Laboratory

1998

\section{Overview and Goals}

This project is expanding the present NIST Special Test Service for digital multimeters (DMMs) and multifunction calibrators, used to test DMMs for the five electrical quantities they normally measure-ac and dc voltage and current and dc resistance. The expanded service is similar to Measurement Assurance Programs (MAPs) that are in place within the Electricity Division, where NIST-owned transportable standards are calibrated and sent to customers to calibrate their equipment. Also, the SIMNet activities described in the Program Accomplishments section of this document have been performed in conjunction with this project.

The developed service enhances communication between NIST and its partners using the Internet. The instrumentation tested is owned by each of the partners and, except for occasional calibration at NIST, remains at the partner's laboratory. Like a MAP, NIST characterizes the transportable standard and analyzes the test data. In addition, NIST can control parts of the test remotely to assure that the measurements at the partner's laboratory are in control. The extent of the value and cost effectiveness of this new type of service is being evaluated to determine the potential for it leading to a future NIST interactive Calibration Service.

Several models of DMMs and multifunction calibrators are characterized using the NIST Multifunction Calibration System in the Electronic Instrumentation and Metrology Group in the Electricity Division. Other projects in this group include Measurements for Complex Electronic Systems and Strategies for Testing SoftwareEmbedded Systems. Expertise from these areas has been utilized to develop instrument models that can estimate the influences of environmental and signal parameters for each instrument type. Measurements are performed to determine parameter coefficients for each class of instrument and the extent to which these parameter influences interact. Correction algorithms optimize performance. Software developed for use through Internet connections permits either a DMM or calibrator to be used as a reference to calibrate other instruments at the partner's laboratory.

\section{Initial Comparison of Standards}

To facilitate standards comparison, a test system consisting of a DMM, multifunction calibrator, environmental monitors, controlling software, computer, and the audio/video link, is set up at NIST and the partner's laboratories. A NIST-calibrated DMM is used to calibrate a partner's multifunction calibrator, using their normal test procedures. The same instrument is then calibrated using NIST procedures and software operating through an Internet connection. Test parameters are monitored during both tests to optimize the DMM performance, and the results obtained from 
these partner- and NIST-controlled tests can be compared and significant differences resolved. This is the most important step in the process, where the partner's test procedures can be evaluated more thoroughly than a conventional MAP allows.

\section{Level I monitor}

A monitor program is established once the measurement systems have been analyzed and normalized to yield consistent results. Tests can then be performed, under NIST control, to verify that the DMM and calibrator are within the desired tolerance. Measurements also provide new data for instrument models, which can be used to predict when out of tolerance' conditions are likely to occur, requiring recalibration at NIST.

\section{Level II monitor}

To verify lower level measurements (like those made by the partners to support their manufacturing processes), an instrument from their workload can be tested using NIST test procedures. This test can be compared to the most recent test performed by the partner to identify possible problems in the test procedure and to verify assigned uncertainties. This verification process can be used to confirm test procedures and standards at every echelon in the partner's measurement chain.

The NIST tests will be operated through a high speed communications line providing an audio/video link for real-time trouble shooting and consultation, and a data link permitting rapid data analysis. Once proven, the procedure will be opened to other interested partners who are willing to invest in the test and communication equipment. Most customers who might use this service already own the necessary test equipment. The communications equipment will be the major hardware expense. However, this impediment should disappear with time as improvements are made in standard Internet capabilities.

\section{Benefits}

As the number of calibration systems grows, a database that better determines the long-term performance of various hardware/software systems is being developed. A network of 30 to 40 partners with similar test systems that are periodically monitored by NIST staff (through an Internet connection) to determine the health of each system relative to the network is possible. With password access, comparison of customers instrument performance to others within the network can occur (without identifying their owners). Carried further, the database could be opened to all customers of instruments and standards tested at NIST. Done properly, this concept could expand the NIST scope and value to U.S. industry without compromising NIST's impartial role in this measurement arena. 
PROJECT TITLE:

LEAD NIST OU: YEAR ESTABLISHED:
Use of Next Generation Internet and Intelligent Collaboration and Visualization Technologies to Build NAMT Collaboratories Information Technology Laboratory 1998

\section{Overview}

The NAMT has been created as a distributed facility that can be shared with industry, academia, and other government agencies, and within NIST. The NAMT enables the operation of an information-based collaboratory that facilitates joint research, training, and demonstrations among NIST and its partners and customers. The testbed computing and communications infrastructure is central to NIST's ability to externalize and leverage its unique resources and capabilities through the Information-Based Manufacturing Program.

The computing and communications infrastructure of the NAMT has successfully supported full collaboration and access to resources in various capacities for the collaboratory both within NIST and among non-NIST partner organizations. The capabilities of this collaboratory are also evolving toward new communications technologies and computing environments that are emerging out of the Internet networking and collaboration research communities. Details of the technologies of the NAMT, including asynchronous transfer mode (ATM) networking which forms the foundation of the NAMT network backbone, can be found in [1].

Experience with the NAMT has led to the following conclusions:

- Capabilities based upon native ATM tend to be limited to single sites. ATM as a technology and ATM infrastructures and services must make very significant advances before ubiquitous interconnection of native ATM devices is possible.

- Advances in switching and routing have demonstrated that LAN switching and IP routing technologies are capable of operating at speeds comparable to ATM at potentially much lower costs.

- Even if ATM remains a viable layer 2 technology on campuses and within wide area networks, the ubiquitous use of Internet Protocols (IP) and advances in IP performance and services are likely to hinder the desire to use ATM end-to-end. The focus of the evolution of government and academic research networks is on internetworking technology (i.e., IP).

- Speed alone is not "quality of service", (QoS) although it is often assumed to be. Most of today's "challenging applications" could be accommodated by $10 \mathrm{Mbs}$, or certainly $100 \mathrm{Mbs}$, technologies. The difficult issues of performance QoS surround the allocation of resources on communication components that are shared and over committed. In addition, the support of such services as multicast, security, and real-time transport protocols are vital to the support of future applications. Most of the significant advances in these other aspects of QoS have occurred in Internet technology. 
- A focus on developing a portable, potentially publicly available, teleconferencing environment for the Information-Based Manufacturing Program collaboratory greatly enhances the potential for collaboration outside of NIST.

- To facilitate development of collaboratories, computing and communication functionality and tools cannot be vertical/stand-alone. Collaborative manufacturing applications require the integration of audio/video transport, shared workspaces, distributed objects, archival and playback functions, floor control, and multicast data in many and varied ways. Evolving toward a networking/application environment that supports the composition of common components greatly facilitates rapid prototyping of collaborative applications.

The ITL Advanced Networking Technologies Division (ANTD) is involved in several ongoing projects in Next Generation Internet (NGI) and Intelligent Collaboration and Visualization (ICV) technologies. These projects focus on topics that include:

- protocols and architectures for provision of QoS controlled IP services

- end-to-end network security technology and security management protocols

- architectures for IP and ATM integration

- development of NGI testbeds and wide area network connectivity to advanced research networks

- encoding, transmission, and synchronization of multi-media data streams

- test and measurement tools for advanced Internet and ATM protocols

- testing and evaluation of ICV collaboration tools / environments

- development of collaboration tools / environments

While the ANTD is working productively in these areas, it lacks significant applications of its own for these technologies. The availability of a challenging target networking and application environment provides a valuable testbed and goal for several of these efforts. The collaboratory enabled by the NAMT represents such an environment. By collaborating with the NAMT projects and goals, the ANTD is gaining a valuable local testbed for its networking research.

Goals

There is a natural synergy between many of the research issues that the ANTD is pursuing and the long-term goals of the projects of the Information-Based Manufacturing Program. This project is leveraging this natural synergy between the NAMT computing and communications effort and the ANTD efforts in NGI and ICV technologies. This project is:

- establishing a distributed NGI and ICV testbed in conjunction with wide area NGI testbed activities

- focusing on manufacturing applications in the evaluation of $\mathrm{NGI}$ and ICV technologies 
- prototyping and evaluating advanced Internet technology and ICV tools to replicate collaboration capabilities enabled by the NAMT, but making them available to a much wider community

The following outlines the technical approach.

- NGI Testbed - A distributed NGI testbed has been established. This testbed is interconnected with other federal NGI research networks.

- NGI Test and Measurement Technologies - Application benchmark suites have been developed in cooperation with NASA, focusing on manufacturing applications in developing requirement characterizations and testing methodologies. ANTD tools measure and analyze the QoS requirements of NAMT applications, and distributed test tools are capable of executing benchmark suites on technology under evaluation.

- Evaluation of NGI Networking Technologies - In cooperation with NASA and several potential vendors of advanced Internet technologies, the project is conducting testbed deployments and evaluations of advanced IP switching and routing technologies and IP/ATM integration architectures. Evaluation focuses on the performance/scaling requirements for manufacturing applications using NIST developed test and measurement tools to support evaluations.

- Pilot Deployment and Evaluation of ICV Technologies - A prototype ICV software infrastructure is being deployed based upon the University of California at Berkeley's Multimedia Architecture that Scales across Heterogeneous environments (MASH) toolkit, replicating NAMT-enabled collaborative applications using advanced IP and ICV technology. NAMT-enabled applications in local and wide area research network environments are being tested and evaluated in both workstation and Windows PC environments. Methodologies and tools to instrument collaborations based upon the MASH Toolkit are being developed and tested, and these tools are being used to characterize the resource requirements and usage profiles of manufacturing collaborative applications.

- Prototype Development / Deployment of Advanced Collaborations - The MASH Toolkit is being used as the foundation to prototype / deploy more advanced collaboration capabilities for NAMT-enabled applications.

\section{Benefits}

The Information Based Manufacturing Program has been working to direct its future computing and communication infrastructure in a direction that is in line with the broader NGI and collaboration research communities. This project has resulted in prototype collaborative manufacturing applications that readily permit collaboration with industry and academia over tomorrow's commercial Internet. The ITL ANTD has, as a result of this project, the availability of the Information-Based Manufacturing Program collaboratory applications and networking environment as a means for furthering research in this area. 
PROJECT TITLE:

LEAD NIST OU: YEAR ESTABLISHED:
Verification of IT Solutions for Small and Medium Size Manufacturing Enterprises

Manufacturing Extension Partnership

1998

\section{Overview}

Large US manufacturers are among the world leaders in the application of information technologies. Studies and focus groups suggest that while small and medium size manufacturing enterprises (SMEs) are investing large amounts of capital in IT consulting services, they're also purchasing piecemeal applications that are inappropriately scaled for their businesses. To compound the problem, SMEs are not training their staffs appropriately to use the IT solutions in which they are investing. Many IT hardware and software solutions sit idle, are used inappropriately, or are not used to their maximum advantage.

Large firms have acquired IT implementation expertise through investments in internal IT staffs in conjunction with obtaining development and integration services of large national consulting firms and IT vendors. These avenues are typically not affordable to SMEs, who turn to local consultants and Value Added Resellers (VARs) for their IT solutions. These providers are generally less capable of providing the level of expertise required to integrate and support an efficient IT solution for the SME. Overall, SMEs are successfully implementing information technologies more slowly than large firms. This is contributing to an increasing disadvantage in the SMEs competitiveness in the global marketplace.

Finding a solution to this problem is critically important to the competitiveness of U.S. SMEs for the following reasons:

1. Lower trade barriers and the explosion of internet technologies have accelerated international competition, and supply chains of large OEMs are extending international competition to U.S. SMEs occupying the lower and mid regions of the supply chains.

2. Companies that can not effectively utilize information technology in a supply chain environment will be less able to participate in major sectors of manufacturing.

3. Foreign trading partners provide their SMEs with extensive support to organize and use information technology.

4. Manufacturing is extremely competitive. Companies that do not perform well in the areas of price, quality, timeliness, service and innovation will fail. Effective application of information technology in the SME environment can

- help lower the SME's costs

- help the SME improve the timeliness of product development and delivery to the marketplace 
- help the SME enhance product quality

- help the SME innovate its products and processes

Without the successful integration of new information technology, many U.S. small and medium-sized manufacturers may be forced out of business as supply chain integration and electronic commerce extend world wide through Internet-based technologies. The vast majority of SMEs attempt to access new information technologies through a bewildering, complex and unstable marketplace. Unlike large manufacturers SMEs cannot afford to hire internal IT staff, national consultants, or turn to large vendors for advice.

The growing information technology workforce shortage, being cited by many as reaching national crisis proportions, is making it even more difficult for SMEs to obtain IT skilled workers. It is risky for SMEs to hire vendors or VARs for fear of having their product lines exclusively imposed, which may not be the right solution for the SME's business. The inability of the SMEs to obtain an impartial recommendation from a knowledgeable source in which they can trust results in high risks and many times diminished benefits to their businesses.

\section{Goals and Benefits}

The situation just described creates the need for the NIST MEP to offer unbiased and well tested IT services on which SMEs can rely. MEP centers can provide an impartial assessment of product offerings best suited for a given SME's manufacturing environment, and assist in delivering the appropriate integration and support services. There is a wealth of knowledge and expertise pertaining to manufacturing enterprises within the NIST labs that can be of great benefit to MEP in defining state of the art information technology solutions for SMEs.

This project leverages the NAMT computing and communications infrastructure to draw upon this expertise and support the piloting of information technology solutions within operational scenarios with small manufacturers to verify the suitability and effectiveness of those solutions within the SME environment.

This project provides the capability to demonstrate performance efficiencies that SMEs can achieve through the application of new IT solutions. It provides the basis for the NIST MEP to develop IT product and service guidelines for MEP centers. The project is developing an ongoing testing capability to pilot prototype configurations of maturing information technologies from the NIST laboratories within a collaboratory environment to verify their maturity and readiness for deployment by the MEP system. This testing also provides a pipeline for feeding industry requirements, especially those from the SME sector, back to the NIST laboratories to help focus research activities on industry needs. 
PROJECT TITLE:

LEAD NIST OU:

YEAR ESTABLISHED:
Interface Standards and Internet Technologies for Robotic Arc Welding

Materials Science and Engineering Laboratory, Boulder, Colorado

1998

\section{Overview}

This project is addressing issues facing the rapidly growing and developing robotic welding industry. An industrial panel at an industry workshop held at NIST in 1995 strongly recommended that NIST help develop interface standards and data exchange standards between the various components in a robotic welding cell. The problems encountered in integrating many specialized components are limiting the range of applications that can benefit from automated arc welding.

Most manufacturers tend to buy a welding cell from a particular integrator. The integrator delivers a welding power supply, robotic arm, part actuator, a programmable system controller, and, occasionally, a process sensor. The interfaces between the programmable system controller and the other components of the welding cell are usually proprietary, forcing the user of the welding cell to make future purchases only from that integrator. Users usually choose the best technology that is available at the time of the purchase of welding cells for a specific welding operation. This often leads to a number of different kinds of power supplies and robot arms, each with its own controller. This multiplicity leads to additional training and maintenance costs. Further, lack of standard interfaces make it difficult for suppliers of innovative sensors to have their products integrated into welding cells.

Many manufacturers that use robotic-arc welding cells have plants across the nation. The welding engineers in one plant often face the same problems as the engineers in another plant. Effective communication about the problems usually requires travel by one or more of the engineers. Also, when a new part is to be welded, the welding engineer picks the process variables usually through experiments in the welding laboratory. Transferring the optimal process parameters to the production floor is not always straightforward because of differences between the equipment on the floor and in the lab (again the need for interface and data standards). If the development engineer can remotely access the welding cell on the manufacturing floor while developing the process in the laboratory, setup costs can be reduced and time to tune the process would be shortened.

Most assembly lines in this country do not produce a quantitative measure of the quality of the parts that are being manufactured on a part-by-part basis. This is true of the robotic welding assembly lines. If quality data is collected, it is often a laborious process that can consider only a small number of parts. If a welding cell is collecting quality information via process sensors on every part, it only makes sense that this be made available in the most convenient format possible. There is no appropriate mechanism, however, to get this information to the engineers; therefore, valuable 
data on the process quality (generated in real-time as the products are being produced) is generally not being used.

Goals and Benefits

The goals of this project are:

- to develop and validate interface standards for robotic weld equipment

- to use Internet technologies to allow for collaboration in weld cell programming, remote welding, and process monitoring

- to develop and demonstrate an intelligent welding cell that can be used as a national test bed for sensor developers, arc welding power supply manufacturers, and robotic arm makers

In addition to interface and data standards, some specific needs also being addressed are:

- better communication between production control and the shop floor

- better programming tools (including simulation)

- better sensors for the welding process

- ease of transferring data and remote access to control processes

- the availability of welding quality data

- performance of real-time process controllers

The computing and communications infrastructure provided by NAMT, especially the high-performance network, has been key to the success of this effort. 
PROJECT TITLE:

LEAD NIST OU: YEAR ESTABLISHED:
Internet-Based Calibration Services for Radiation Processing Industries

Physics Laboratory

1998

\section{Overview}

NIST employs the alanine-electron paramagnetic resonance (EPR) dosimetry technique for calibrating industrial radiation sources. This technique is widely recognized as the best of the available methods for relative high-dose measurements and is currently used by not only NIST, but also all major international metrology laboratories. This technique is based on relative EPR measurements of the concentrations of stable free radicals induced by radiation in alanine. The concentration is unambiguously related to the absorbed dose. Therefore, it is possible to assess the absorbed dose of a test alanine pellet by comparing the intensity of its EPR signal with the intensities of the same signals from identical pellets irradiated to precisely known doses with a standard source.

Very high precision is achieved by EPR; the overall uncertainty of this dosimetry method is less than $2 \%(27)$ [12]. In combination with the extremely wide range of doses covered by this technique, independence of the results of dose rate, operation simplicity, and a number of other favorable features, this high accuracy makes the alanine-EPR method unparalleled in high-dose radiation measurements.

\section{Goals and Benefits}

The objective of this project has been the creation of a system to be used for NIST to provide fast, remote calibration services of high-dose radiation sources against the U.S. national standard gamma-radiation source using the Internet. The Internetbased system can deliver immediate calibration results to the industry customer ondemand at a lower cost than the current NIST service. The calibration results can be rapidly incorporated into the product dose-map model to ensure the highest quality manufacturing.

The typical procedure for calibrating a source for a customer comprises sending unirradiated alanine pellets from NIST to the customer, who irradiates them with the industrial radiation source to be calibrated. The irradiated pellets are returned to NIST, the EPR signals are measured at NIST, compared with the signals from pellets irradiated with the NIST standard calibration source, and the dose values are calculated. Finally, a NIST Certificate of Calibration containing the NIST-interpolated dose values is sent to the customer. This process takes several days and considerable labor at NIST, which makes the cost of the calibration relatively high and prevents customers from frequent use of this NIST service. In some cases, U.S. regulatory agencies require only that the industrial process be tied to a national standard, not the U.S. (NIST) standard. Consequently, many U.S. companies have 
sought to reduce their cost by using foreign calibrations, since the turnaround times are similar.

Automating this operation on the basis of modern technologies and commercially available products is an integral part of this project. Relatively small EPR spectrometers are available commercially that are designed specifically for dosimetry purposes. These instruments can exhibit the necessary high accuracy of measurements in combination with a significantly reduced cost. These spectrometers can be used in parallel at a customer's laboratory and at NIST for concerted measurements. High-quality alanine pellets are also available commercially. These can be used for the calibrations in question and are available both for the interested customers and NIST directly from the company that manufactures them. Finally, there is the Internet, which provides an easy link between the EPR spectrometer and computers at NIST and the identical computer-controlled EPR spectrometer at the customer's laboratory. The entire redesign of the source calibration process by combining these means is a focus of this project. This will make the process faster, less laborious, much cheaper and, consequently, much more accessible for present and potential customers.

The Internet-based transfer calibration process being developed in this project can be described as follows. The industrial site is first be connected to the NIST calibration facility via Internet and fiber optic link. The industrial facility accesses the Internet program, requests a calibration, and begins by irradiating pre-supplied dosimeters. The Internet calibration program controls their EPR spectrometer and instructs the industry technician to perform the required measurements in a step-wise manner. The raw data is transferred and evaluated by the program and a provisional certificate of calibration is generated for the customer. The clear advantage is that these calibration data can be used immediately in the industrial process. After appropriate quality checks are made at NIST, an official certificate is issued (this step is to fulfill the regulatory requirement of a signed calibration certificate, and does not slow the application of the calibration data by the customer). The Internet calibration program provides industry with on-demand calibrations, immediate turnaround times, lower cost, and improves the quality of the manufacturing process.

The Internet-based calibration service is being established in a series of progressive stages. The first stage has been the establishment of a testbed system within NIST. This system is built in consultation with industrial partners to facilitate its immediate implementation upon transferal. Radiation processing facilities operate non-stop and do not have the luxury to test and develop dosimetry systems. A fully-functioning testbed system can be used to demonstrate and train industry partners. The system is being provided to companies representing each of the three major high-dose radiation applications: food irradiation, medical device sterilization, and materials processing. 


\section{REFERENCES}

1. "National Advanced Manufacturing Testbed," Stieren, D., Densock, R., and Luce, M., NISTIR 6383, U.S. Department of Commerce National Institute of Standards and Technology, Gaithersburg, MD, August 1999.

2. National Collaboratories: Applying Information Technology for Scientific Research, Committee on a National Collaboratory, National Research Council Commission on Physical Sciences, Mathematics, and Applications, National Academy Press, Washington, D.C., 1993.

3. SIMnet Design and Internet Deployment Guide," Schneeman, R., NISTIR 6452, U.S. Department of Commerce National Institute of Standards and Technology, Gaithersburg, MD, December 1999.

4. Methods for Performance Evaluation of Computer Numerically Controlled Machining Centers, American Society of Mechanical Engineers Standard B5.54, New York, New York, 1992, reaffirmed 1998.

5. Test Code for Machine Tools, ISO Standard 230-1, American National Standards Institute, New York, New York, 1996.

6. "Enhanced Machine Controller Program Summary," U.S. Department of Commerce National Institute of Standards and Technology, Manufacturing Engineering Laboratory, http://www. mel.nist gov/projs/isd/emc.html

7. "NIST Construction Metrology and Automation Program," presentation given to Program Managers of the National Science Foundation by Dr. William Stone, U.S. Department of Commerce National Institute of Standards and Technology Building and Fire Research Laboratory, Gaithersburg, MD, March 1999.

8. "Virtual Die Design for Information-Based Metal Forming," presentation given to the Director and Deputy Director of the National Institute of Standards and Technology by Dr. Timothy Foecke, U.S. Department of Commerce National Institute of Standards and Technology, Gaithersburg, MD, November 1997.

9. "Design, Manufacture, and Calibration of Brachytherapy Sources," presentation given to the Director and Deputy Director of the National Institute of Standards and Technology, by Dr. Christopher Soares, U.S. Department of Commerce National Institute of Standards and Technology, Gaithersburg, MD, November 1997.

10. "Building and Fire Research Laboratory Research Update," U.S. Department of Commerce National Institute of Standards and Technology, Gaithersburg, MD, Volume 3 Number 3-4, Winter 1998, http://www.bfrl.nist.gov/bfrlnews/v3n3.html 
11. "Tele-Calibration of Gas Flow Meters, Espina, P., 1999 NCSL Workshop and Symposium Proceedings, National Conference of Standards Laboratories, Boulder, CO, 1999.

12. "Remote Calibration Services for the Radiation Processing Industry," presentation given to the Director and Deputy Director of the National Institute of Standards and Technology, by Dr. Marc Desrosiers, U.S. Department of Commerce National Institute of Standards and Technology, Gaithersburg, MD, February 1999. 

